\title{
Production Capacity Investment with Data Updates
}

\author{
Philip Kaminsky · Ming Yuen \\ Dept. of Industrial Engineering and Operations Research \\ University of California, Berkeley, CA 94720
}

September 17th, 2013

\begin{abstract}
We model the capacity investment problem faced by pharmaceutical firms and other firms with long and risky product development cycles. These firms must balance two conflicting objectives: on one hand, the delay in scaling-up production once the product is approved must be minimized, and on the other hand, the risk of investing in ultimately unused capacity must be minimized. We develop and analyze a stylized model of this type of capacity investment problem, where the firm re-evaluates its capacity investment strategy as information about the potential success of the product is continually updated (for example, via clinical trial results in the case of the pharmaceutical industry). Motivated by observations of current practices in the biopharmaceutical industry, we use a computational study to explore how practices such as more frequent reevaluations of investment decisions, stopping and restarting of projects, and the use of alternative types of capacity can, under certain conditions, help the firm reduce both the delay of the commercial launch of the new product, and the risk of lost investment. Supplementary materials are available for this article. Go to the publishers online edition of IIE Transaction to find detailed proofs of the results in this paper.
\end{abstract}

Keywords: Capacity expansion, Pharmaceutical planning, Dynamic programming, Bayesian updating

\section{Introduction}

In industries with long product research, development, trial, or approval times, and extended capacity acquisition times, firms may have incentive to invest in production capacity for a product before learning if that product will ever go to market. In this setting, firms often have to make capacity investment decisions even as they continue to acquire information about whether or not 
this capacity will prove useful. In this paper, we explore this class of capacity investment decisions, and how these decisions are impacted as additional information becomes available. To develop insights, we analyze a model in which in each period firms receive updated information about the likelihood that a product will come to market, while simultaneously making decisions about whether or not to invest in production capacity.

\subsection{Research Goals and Motivation}

Our goal in this paper is twofold: we develop and analyze a series of stylized models to characterize decision making about capacity investments in this setting, and, motivated by our experiences with a major biopharmaceutical firm, we complete a series of computational experiments to explore the effective use this information. Indeed, this research was specifically motivated by a modeling exercise that we observed at this major biopharmaceutical firm (and more generally, by discussions with the members of an NSF-sponsored I/UCRC focusing on Biopharmaceutical Operations that one of this paper's co-authors directs). Due to the high cost of shortages upon drug approval, firms in this industry may have incentive to invest in production capacity for a product before it is known if the product will ever go to market, and thus face risks related to the long horizons for clinical trials, "all-or-nothing" demand for new products depending on the outcome those clinical trials, long lead times for the construction of manufacturing capacity, and extremely high costs for construction of that capacity. Our computational experiments in particular are specifically motivated by issues faced by the biopharmaceutical industry, but the models (as well as some of the computational insights) can apply to any industry with expensive capacity, long research and development time that could potentially end in failure, and long capacity acquisition lead times.

The modeling exercise we observed was used by the firm to help project its long term capacity investments and the timing of those investments, given drugs in various stages of development or testing (known as clinical trials in this industry); the three to five years it would take to acquire, build, or plan for outsourcing appropriate capacity for each product (as estimated by the firm); and the fact that in the biopharmaceutical industry, demand for products is quite well understood given that certain discrete events (such as successful clinical trials) occur. Management was also interested in better understanding the value of an emerging alternate manufacturing approach in this industry, built around smaller scale disposable technology - this approach is known to have a significantly shorter acquisition lead time, but is likely to have a larger total cost of acquisition and

operation over the life of a typical product. We were particularly struck by three characteristics of the approach that this firm used to model future capacity needs - characteristics that managers from 
other firms with whom we've discussed related issues confirm are quite common in the industry: (1) The the firm went through an annual planning process, using point estimates of the likelihood of discrete events (such as successful drug approvals) for several years into the future, in spite of the fact that data is continually collected during ongoing clinical trials, which would allow significantly more frequent updates; (2) the planning process ignores the fact that these estimates are updated, and that the firm's confidence in the estimates of the likelihood of the outcome of these events increases over time as the events get closer; and (3) although capacity investment projects have many steps, including acquisition of equipment, paying of fees, construction, etc, and thus could potentially be paused or halted as the likelihood of events such as product approvals changed, the firm assumed that once a project had started, it would be completed. In the case of the alternate "disposable" production technology mentioned above, it might even make sense to initially invest in the traditional longer construction lead time approach, and then switch to this alternative approach if necessary.

In this paper, we analyze a family of stylized models that begins to explore the possibility and value of relaxing these typical assumptions, and that provides insight into the value of the novel production technology mentioned above. Although the process of developing biopharmaceuticals (and more generally, pharmaceuticals and many other products) is quite complex as we discuss below, we focus on capturing in a tractable way two key characteristics of the problem - an estimate of the likelihood of ultimate approval (that is, the likelihood that the product will actually go on the market) that is updated over time, and a capacity investment that is made over time as this estimate is updated. To do this, we analyze several versions of a discrete time finite horizon model, in which the clinical trial is modeled by a Bernoulli process with unknown rate. In each period, the result of one additional Bernoulli trial is revealed to the firm, and the firm's estimate of that rate is updated. If the number of successful trial results observed by the final period of the horizon exceeds some pre-determined threshold, the product is approved. Simultaneously, in each period the firm must decide whether or not to invest (in that period) in a capacity project, where the completion of a project requires the firm to invest for a pre-specified number of (not necessarily consecutive) periods. If, at the end of the horizon, the product is approved but the capacity is not yet completed, the firm must pay a penalty. If, on the other hand, at the end of the horizon the product is not approved but the firm has invested, that investment is lost. 


\subsection{Background}

\subsubsection{Clinical Trials}

Of course, this is a highly stylized model of the clinical trial process. In the biopharmaceutical industry, for example, introducing a new drug is a complex and time consuming process. Research and development (R\&D) alone is risky, expensive, and time consuming. After discovering a drug, the firm has to demonstrate safety and efficacy of the new drug for a particular disease or set of symptoms to regulatory agencies via a series of clinical trials in order to gain the approval to put the new drug on the market. Typical trials have three phases: the first two involve testing for safety and effectiveness on small groups of patients, while the third involves large sample size statistical studies (often involving thousands or tens of thousands of patients) either comparing the performance of the new drug with an existing treatment, or with no treatment.

Phase III drug trials are often very complex, ranging from trials that compare the performance of the new drug to some historical data, to randomized double blind trials with a placebo or alternative treatment, to so-called paired outcome and "cross-over" designs, where patients switch treatments at some prespecified time, to factorial designs where several potential treatments are compared (Friedman et al. (2010)). Response variables can range from binary (the drug either works or does not, the patient dies or does not) to continuous measures of efficacy. These trials require sophisticated statistical analysis, where both regulatory agencies and the firm pay close attention to controlling type I and type II error. Indeed, clinical trials can often cost millions of dollars, and on average it takes eight years for all of the clinical trials to be completed and a drug to be approved (DiMasi et al. (2003)). Our Bernoulli model of clinical trials (which can be viewed in the language of clinical trials as a simple confirmatory trial with a historical control) obviously does not capture all of the details of many trials. It ignores, for instance, randomized testing, controls, continuous measures of drug efficacy, etc. However, our model enables us to capture in a relatively straightforward and analytically tractable way the key characteristics of clinical trials most relevant to addressing the questions raised above, including the updating of results over time, predetermined sample sizes, and success thresholds designed to control statistical error.

\subsubsection{Capacity Expansion}

Our model of capacity investment is similarly stylized. Typically, the construction or acquisition and licensing of physical production capacity for a new biopharmaceutical is an expensive and 
time consuming project. Although the firm requires some production capacity to support clinical testing, this capacity is typically not sufficient to meet demand once a drug is approved. Building and licensing a new traditional commercial-scale capacity can take 4-5 years and cost up to $\$ 800$ Million US (see, e.g., Genentech (2009), Snow et al. (2005)). Even if a firm plans to produce the drug in an existing facility or outsource the product to a contract manufacturing organization, it typically takes several years and costs $\$ 100$ 's of millions to make necessary modifications, develop an effective approach for technology transfer, and complete FDA licensing (Snow et al. (2005)). Because biopharmaceuticals are difficult to analytically characterize, biopharmaceutical licensing is tied to the facility in which the product is made. Thus, changing or adding to manufacturing capacity after a product is licensed is expensive and risky, so firms typically focus on having sufficient production capacity to meet future demand available at a single site at product launch, and are hesitant to change or expand capacity after that point.

Recently, novel so-called "disposable" or "single-use" approaches to biopharmaceutical production utilizing, for example, one-time-use tank liners and mixers, have become viable options, at least at a relatively small scale (Morrow (2006), Xcellerex (2012)). These options take less time to build and install than traditional large stainless-steel tanks and processing equipment, and may allow for rapid ramp-up of capacity, as they are typically pre-validated, pre-sterilized, and stocked by vendors. However, they are smaller scale than the traditional "stainless-steel" capacity used by major biopharmaceutical firms (see, e.g., Snow et al. (2005)), and thus the appropriate strategy for implementing these technologies is still under consideration at many firms in the industry. Since this capacity by definition has to be replaced each time it is used, it is likely that operating costs will be significantly higher, although the capacity installation costs relative to traditional approaches remains ambiguous.

As with our model of clinical trial updates, our model of capacity expansion ignores many of the complexities of this capacity expansion process in the interest of analytical tractability, but allows us to capture the characteristics of the process most relevant to the questions raised above: the long lead time inherent in biopharmaceutical capacity expansion, and the fact that the firm has the option to abandon or pause investment in a capacity expansion project at several points over the horizon. In reality, the firm typically doesn't consider a single product in isolation - the firm is typically developing a portfolio of products, and may have a portfolio of capacity investment projects. Explicitly capturing these portfolio effects would require more elaborate models than we consider here, and thus we do not capture the risk pooling inherent in portfolios of products and capacity. However, even in this case, as we alluded to above, capacity ultimately needs to be 
modified and licensed for specific products using specific technologies, a process that is expensive and time consuming, and requires differentiation for specific products.

\subsubsection{Motivation}

The biopharmaceutical firms with which we've worked typically commit to building or acquiring production capacity early enough in the trial process to ensure that this capacity will be ready at the time that the drug receives final approval. Of course, there is no guarantee that a new drug will pass all of the required phases of clinical trials, and in fact most drugs fail to show effectiveness at Phase II and Phase III of clinical studies. For example, consider a few randomly selected headlines from late May and early June of 2012:

- In fresh setback, Alnylam's most advanced drug flunks PhIIb RSV test (Carroll (2012a))

- Amgen's Mimpara disappoints in phase III study (PMLive (2012))

- Repligen shares slide after FDA rejects imaging agent (Carroll (2012b))

Thus, by committing to building or investing in a facility without carefully assessing the appropriate level of evidence that a drug will pass the required clinical trials, and by not updating this decision as information becomes available, the firm may be taking on excessive investment risk. In this paper, for reasons of tractability, we assume that if a drug fails clinical trials, the entire capacity investment is lost. In practice, firms may adapt partially completed investments for other pipeline products, but as we observed above, the process of adapting an existing facility for a new product is by itself very expensive and time-consuming. On the other hand, a firm has a limited window of exclusive sales rights for any drug before generic drug-makers enter the market and drive down prices, and this is typically the firm's primary opportunity to recover enormous initial investment costs. In the US, for example, biotechnology firms have twelve years of exclusive sales of a drug before generic versions of a product can be introduced (Gayle (2010)), and similar regulations apply in other parts of the world. Thus, if the firm underestimates the likelihood of approval, it may act too conservatively when making production capacity investment decisions, and thus be unable to take full advantage of its limited window of exclusivity. Perhaps more importantly, patients will suffer if the availability of safe and approved drugs is delayed.

Our goals in this paper are to analyze effective decision making in the context of the stylized model described above, and to complete a series of computational experiments to explore if and when it is useful to relax the three typical assumptions mentioned above when planning capacity investments in this setting and to explore the impact of the availability of emerging single-use-based capacity on capacity planning. In particular, we address questions such as: When is it valuable to 
frequently update estimates of the likelihood of a successful clinical trial? How frequently should these estimates be updated? What is the value of explicitly modeling the process by which these estimates are updated, so decisions account for the fact that information will be more precise in the future? What is the value of regularly reconsidering capacity investment decisions? How does the availability of an alternative, more expensive, shorter lead time project impact these observations?

To do this, we characterize the optimal investment strategy for several versions of the model discussed above, then complete a computational study to develop additional insights into the questions raised above.

\section{Literature Review}

Models intended to determine when, how much and what kind of production capacity to build can be found in the literature as early as the 1960's. Since then, a rich body of research focusing on capacity planning and investment problems under various settings has developed, focusing on issues including demand risk, production and capacity installation leadtimes, and product life cycle. Luss (1982) and Van Mieghem (2003) provide comprehensive surveys of the capacity expansion literature. By assuming a deterministic growth rate of product demand, early work by Sinden (1960), Erlenkotter (1977) and Smith (1979) models production capacity as a type of inventory and approaches the problem with EOQ-like techniques in an infinite horizon setting. Although these technique effectively capture the trade-offs between backlogging and capital costs, these deterministic models fail to address demand risks that manufacturers often face in capacity planning.

Manne (1961) was among the first authors we are aware of to incorporate the notion of demand risk into the capacity expansion literature by explicitly modeling stochastic demand. By considering a particular class of Markov steady state demand growth model and no lead time for building capacity, this paper (as well as those by subsequent authors such as Bean et al. (1992)) develops deterministic equivalents to the stochastic models. Explicit consideration of lead times significantly increases complexity of these models. Elsanosi et al. (2000) and Ryan (2004), in particular, use optimal stopping time models with appropriate adjustments to model capacity acquisition with lead times in a stochastic setting. Davis et al. (1987) utilize a stochastic control model, where the firm continuously controls the amount to invest in building capacity to meet stochastic demand with random lead time. A related stream of literature uses real options analysis to model the risk of making advance decisions due to procurement or production leadtimes. Real options models analyze and evaluate capital investment projects by using stochastic control techniques developed 
in the financial options literature; Dixit and Pindyck (2004) and Trigeorgis (1996) offer extensive outlines of this topic. This literature typically assumes a stationary underlying stochastic process, however, and a key feature of our model is the improving quality of the estimate of the likelihood of approval increases over time.

Several recent streams of research focus on strategies such capacity procurement contracts, capacity outsourcing, capacity pooling, and capacity outsourcing to mitigate capacity demand risk (see, for example, Plambeck and Taylor (2005), Van Mieghem (1999), Chod and Rudi (2006), and Chao et al. (2009)). Although biopharmaceutical firms do outsource certain production, as discussed above the cost and time involved with preparing contract manufacturing facilities for new products are significant, but are not modeled in the existing literature. These sorts of contract manufacturing agreements can be captured by the capacity investments in our model. In addition, many biopharmaceutical firms are concerned about the intellectual property implications of outsourcing manufacturing, particularly because there is a good deal of ambiguity about the nature of intellectual property in biotechnology (Gold et al. (2002)).

The multi-armed bandit problem originally introduced by Robbins (1952) is often used to model the choice among the bets with unknown odds to maximize profits or among R\&D projects with known success rates to maximize potential returns. In this setting, the firm must sequentially select among several experiment types, each with an unknown probability of success, with the long run goal of maximizing the number of successes. After each experiment, the firm updates its estimate of the success rate of that experiment type. Although Bayesian updating of the unknown parameters of the multi-armed bandit models captures the notion of updating key estimates used to select investments, these bandit models generally do not capture the complexities inherent in ensuring that capacity is ready for commercial launch of the product.

The inventory models described in Scarf (1959), Azoury (1985) and Lariviere and Porteus (1999) also use Bayesian learning to model the progressive improvement over time of the decision maker's knowledge of the underlying stochastic demand. In these discrete time models, the demand in each period is modeled by an independent and identically distributed random variable with unknown parameters. By assuming a class of continuous distributions with known conjugate prior, such as the "newsvendor distributions" described in Braden and Freimer (1991), the decision maker in these models progressively updates the posterior distribution of the unknown parameters using the observed demand in each period, and then uses this information to optimize the inventory strategy to maximize profit over the planning horizon. Although we are also concerned with updating an estimate over time in order to ensure that demand can be met, the nature of the investment risk 
described in our capacity investment problem is very different than that of the inventory models. In contrast to the setting in those papers, we explicitly consider the multiple investments over time necessary to acquire production capacity, and "all-or-nothing" demand depending on the success of the clinical trial.

A variety of authors have explored the use of mathematical programming models for the complex planning and scheduling problems encountered in the pharmaceutical manufacturing. Papageorgiou et al. (2001), for example, models pharmaceutical operations at a high level, jointly optimizing production management and financial decisions. Lakhdar et al. (2005) solves a pharmaceutical production scheduling problem in a multi-product and multi-facility setting. Both of these papers address capacity planning, and in particular, Farid et al. (2005) assumes flexible capacity in the scheduling model. However, R\&D-related information is not a focus of these models. Other solution approaches are employed elsewhere in the pharmaceutical capacity planning literature. Farid et al. (2005) uses Monte Carlo simulation to compare the cost of traditional large stainless-steel tanks and single-use equipment for manufacturing clinical trial materials. George et al. (2007) uses expected net present value analysis to compare a series of potential investment options: partnering with another manufacturer for launching a new drug, using a contract manufacturer, or building a production facility from scratch. Although these models account for the risk associated with product launch and FDA approval, in contrast to our work they do not explicitly consider information updates from ongoing clinical trials.

Next, we develop a stochastic process to model the updated estimate of the likelihood that the new drug is approved, and define an associated dynamic programming model to model the capital investment decisions involved with building production capacity for the new drug.

\section{The Model}

We consider a stylized model of a firm that receives a stream of information updates while making a series of capacity investment decisions. The model applies to any firm that completes a series of experiments to determine if a new product should be introduced, but in light of our motivating problem we refer to these tests as experiments or test cases that make up clinical trials, and the product as a drug. (Specifically, we can think of each experiment as the evaluation of the effectiveness of a drug on a single patient.) We assume that there is essentially one (or one remaining) type or phase of trial, made up of a series of independent tests or experiments with the same unknown success rate. A firm must complete this series of tests over a time horizon with $T$ discrete periods 
in order to determine the effectiveness of a product. At the start of period $T+1$, if the clinical trial sufficiently demonstrates the efficacy of the drug, the firm has to produce to satisfy demand, which we assume to be a known constant rate $D$ per unit time since our goal here is to focus on the pre-approval capacity acquisition process. The firm's goal is to build sufficient production capacity to support this demand rate $D$ if the drug is proven effective. If the clinical trial is not successful, the product will not be produced and the production capacity is not needed. We assume building sufficient capacity takes an exogenously determined number of periods, that the firm can revisit its capacity building decision at the start of each period, and that the result of a single case or experiment is revealed at the end of each of the first $T$ periods.

We model the outcome of clinical cases or experiments as independent identical Bernoulli random variables. We motivate this by observing that before a new drug can be marketed, its manufacturer must demonstrate that it is safe and effective via clinical trials or tests on individual patients. We let $p$ be the probability that the drug successfully treats an individual patient in the trial, so that each result represents the outcome of testing on a specific patient. We assume that the Bernoulli rate $p$ is unknown to the firm. This differs from typical assumptions in the real options and capacity investment literature, but better captures the nature of uncertainty in clinical trials. In our model, instead of using a fixed estimate of the unknown $p$ throughout the capacity planning process, the firm updates the estimate of $p$ as the trial progresses.

Regulatory agencies typically require a conventional frequentist approach to assessing the safety and efficacy of new drugs. Nevertheless, the firm typically has access to a large amount of preliminary data (from lab results, early trial phases, expert opinions, etc.), which it has the freedom to incorporate into its decision-making process when it makes other decisions that depend on clinical trial results. In particular, to make capacity-related decisions, the firm is free to use a Bayesian approach that utilizes this preliminary data to estimate a prior distribution of the success rate, and then in each period updates this estimate in the form of a posterior distribution that incorporates new results. Specifically, in our model we specify the underlying stochastic process of our model as follows:

A pair of parameters $\left(\gamma^{0}, \zeta^{0}\right)$ models preliminary data about the potential success of the trial (or more explicitly, about the success rate $p$ ). The prior distribution of the success rate $p$ is thus $\operatorname{Beta}\left(\gamma^{0}, \zeta^{0}\right)$, where $\gamma^{0}=\zeta^{0}=1$ means the firm does not use preliminary data.

At the end of each of the first $T$ periods (after the investment decision is already made for that period), the firm observes an experimental result, represented by the binary random variable $\delta^{t}$, where 1 means a successful experiment, and 0 an unsuccessful one. Thus, the stochastic process 


$$
\gamma^{t-1}:=\gamma^{0}+\sum_{i=1}^{t-1} \delta^{i},
$$

records the number of successful cases observed up to period $t \in\{1,2, \ldots, T\}$ (but not including the result, $\delta^{t}$ ) adjusted for preliminary data by $\gamma^{0}$. The observation $\gamma^{t-1}$ is then the most up-to-date information the firm has when the decision is made for period $t$. We also define $\zeta^{t}$ :

$$
\zeta^{t-1}:=\zeta^{0}+\sum_{i=1}^{t-1}\left(1-\delta^{i}\right)
$$

to count the total number of failed experiments observed (adjusted for preliminary data by adding $\left.\zeta_{0}\right)$, such that

$$
\zeta^{t-1}:=\gamma_{0}+\zeta_{0}+(t-1)-\gamma^{t-1}
$$

Therefore, the posterior distribution of the success rate $p$ at the beginning of period $t$ is $\operatorname{Beta}\left(\gamma^{t-1}, \zeta^{t-1}\right)$. Clearly, the larger the magnitude of the pair $\left(\gamma^{0}, \zeta^{0}\right)$, the stronger the influence of the preliminary data on the estimate of the success rate, $p$, in each future period $t$. In our model, a large $\left(\gamma^{0}, \zeta^{0}\right)$ implies more, and thus higher quality, initial data.

After $T$ periods, the product is considered sufficiently effective if the total number of successful experiments, $\gamma^{T}$, meets the exogenously specified minimum requirement on the total number of successful experiments, $\gamma^{\prime c}$ (so that $\left\{\sum_{i=1}^{T} \delta^{i} \geq \gamma^{\prime c}\right\}$ ). To simplify the exposition, since $\gamma^{0}$ is known, we define $\gamma^{c}:=\gamma^{c}+\gamma^{0}$ so that the event $\left\{\gamma^{T} \geq \gamma^{c}\right\}$ implies the drug has successfully demonstrated safety and efficacy.

In addition, at the start of each period, a capacity investment decision (that is, whether or not to invest in next period) must be made for that period. Once an investment decision is made, the corresponding investment cost is incurred, and one period's worth of construction is completed. In our initial model, we consider a single facility type that requires $s^{0}$ (not necessarily consecutive) time periods to complete construction. The variable $s^{t-1}, t=1,2, \ldots, T+1$ represents the remaining number of periods required to complete the facility at the start of period $t$. Note that $s^{T}$ contains information about how much if any additional time is required to complete capacity at the time when this capacity might be needed. Note that we assume each project can be built within the planning horizon $T$ so that $s^{0} \leq T$.

The cost for one period of construction in period $t$ is $c$. For some of what follows, we consider the impact of a setup cost - an additional cost paid when construction of a facility takes place during period $t$, but did not take place during period $t-1$. If this is the case, the setup cost $K>0$.

At the end of the horizon, in period $T+1$, if the total number of successes $\gamma^{T}$ is less than $\gamma^{c}$, the product is not produced and sold. Otherwise, if the production capacity is not yet ready, the firm 
pays to complete construction, and in addition pays a positive penalty cost $\Pi(s)$ (intended to model the delay in receiving revenue from the product, loss of patent life, loss of revenue, loss of goodwill, etc.) which is a function of the number of periods $s$ required to complete construction. The objective is to develop a capacity investment strategy that minimizes the total sum of discounted expected cost of building the necessary capacity and expected penalty cost.

To model this as a stochastic dynamic program, we number periods forward from 1 to $T+1$. At the start of each period $t, t=1,2, \ldots, T$, the firm observes the remaining construction time for the potential project $s^{t-1}$ (where if the project has not been started, the remaining construction time is equal to the full construction time), the number of successful experiments up to and including the result that arrived at the end of the previous period $\gamma^{t-1}$, and the construction completed in the previous period. To represent the firm's investment decision in period $t-1$, we define $a^{t-1}$ which will equal 1 if an investment was made in the previous period. We assume that the firm has no ongoing capacity investments before period $t=1$, so that $a^{0}=0$. We let $\Omega_{t}, t=1,2, \ldots, T$ denote all admissible investment strategies at time $t$. At the end of the period, after completing construction, the firm observes the next experimental result $\delta^{t}$.

We formulate the dynamic program for periods $t=1,2, \ldots, T$ below:

$$
V_{t}\left(\gamma^{t-1}, s^{t-1}, a^{t-1}\right)=\min _{a^{t} \in \Omega_{t}}\left\{a^{t} c+K\left(a^{t}-a^{t-1}\right)^{+}+\alpha E\left[V_{t+1}\left(\gamma^{t-1}+\delta^{t}, s^{t-1}-a^{t}, a^{t}\right) \mid \gamma^{t-1}\right]\right\},
$$

where $\alpha \in[0,1]$ is the discount factor. In period $T+1$, the firm observes the final experimental result, and thus one of two possible outcomes: either the product is approved and goes into production so the firm pays a penalty if production capacity is not yet ready (if the total number if successes reaches to the threshold value earlier than $\mathrm{T}$, the firm will wait until period $T$ to market the product), or the product is not approved and is not produced. This is captured in the following terminal value function:

where

$$
V_{T+1}\left(\gamma^{T}, s^{T}, a^{T}\right)= \begin{cases}F\left(s^{T}, a^{T}\right) & \text { if } \gamma^{T} \geq \gamma^{c} \\ 0 & \text { otherwise }\end{cases}
$$

$$
F\left(s^{T}, a^{T}\right)=s^{T} c+\Pi\left(s^{T}\right)+K \cdot\left(1-a^{T}\right)^{+} .
$$

In Section 5 of this paper, we characterize the optimal investment strategy in a variety of settings of increasing complexity. In Section 5.1, we consider the base case, where there is no fixed cost (so $K=0$ ). In Section 5.2, we explore the impact of introducing positive setup cost. In both of these sections, we assume a constant per period penalty so that $\Pi(s):=\pi s$. In Section 5.3 , we consider marginal penalty that increases in production delay.

Finally, in Section 5.4, we consider an extension of our basic model where there is an alternative 
capacity investment option that requires a shorter construction leadtime. In this case, in each period we have the option to invest in one of two two capacity types, regular and expedited, where $i \in\{R, E\}$ requires $s_{i}^{0}$ (not necessarily consecutive) time periods to complete construction. Note that although for reasons of tractability we only allow investment in one of the projects in any given period (and based on discussions with managers, given that a firm will ultimately only use one type of capacity when launching a product, it is unlikely that simultaneous investments in both capacity types would be approved), the project selected can change from period to period, so that at any given time both projects can be partially completed. In this extension, we represent the remaining number of time periods to complete the construction as a 2 component vector $\mathbf{s}^{\mathbf{t}-\mathbf{1}}, t=1,2, \ldots, T+1$ where the $i^{\text {th }}$ component represents the remaining number of periods required to complete facility type $i$ at the start of period $t$. (We use boldface to denote vectors, and subscript to denote the components of vectors, so that, for example, $s_{i}^{t}$ is the $i^{t h}$ component of vector $\mathbf{s}^{\mathbf{t}}$.) Therefore, $s_{i}^{0}$ represents the total required construction time for building facility type $i$. Similarly, the cost for one period of construction of a facility type $i \in\{R, E\}$ in period $t$ is $c_{i}$ for each $i \in\{R, E\}$ and $\mathbf{a}^{\mathbf{t}-\mathbf{1}}$ represents the firm's investment decision, where if the firm elects to build facility type $i, i \in\{R, E\}$ at time $t-1$, the $i^{\text {th }}$ component of $\mathbf{a}^{\mathbf{t}-\mathbf{1}}$ will equal 1 ; all other vector components will be 0 . Since we limit investment to one project type in a period, $\mathbf{a}^{\mathbf{t}} \in\{0,1\}^{\{R, E\}}$ and $a_{R}^{t}+a_{E}^{t} \leq 1$ for $t=1,2, \ldots, T$. In principle, we could model setup costs as in (1), but our analysis of the two project model omits setup costs. With this new notation, we formulate the dynamic program for the two-project case as follows:

$$
V_{t}\left(\gamma^{t-1}, \mathbf{s}^{\mathbf{t}-\mathbf{1}}\right)=\min _{\mathbf{a}^{\mathbf{t} \in \Omega_{t}}}\left\{a_{R}^{t} c_{R}+a_{E}^{t} c_{E}+\alpha E\left[V_{t+1}\left(\gamma^{t-1}+\delta^{t}, \mathbf{s}^{\mathbf{t}-\mathbf{1}}-\mathbf{a}^{\mathbf{t}}\right) \mid \gamma^{t-1}\right]\right\},
$$

with terminal value function:

$$
V_{T+1}\left(\gamma^{T}, \mathbf{s}^{\mathbf{T}}\right)= \begin{cases}F\left(\mathbf{s}^{\mathbf{T}}\right) & \text { if } \gamma^{T} \geq \gamma^{c} \\ 0 & \text { otherwise }\end{cases}
$$

where

$$
F\left(\mathbf{s}^{\mathbf{T}}\right)=\min \left\{s_{R}^{T} \cdot\left(\pi+c_{R}\right), s_{E}^{T} \cdot\left(\pi+c_{E}\right)+O\right\} .
$$

Here, $O \geq 0$ is a positive constant that we use to account for potentially higher operating costs of using expedited capacity (as would be expected, for example, if "single-use" capacity is utilized).

In the remainder of this paper, we explore the implications of the firm's capacity investment problem. We begin in the next section with a preliminary analysis of the evolution of the stochastic process $\gamma^{t-1}$. In Section 5, we characterize the optimal investment strategy in a variety of settings of increasing complexity. In Section 6, we utilize a computational study to develop additional insights into appropriate capacity investment strategies for firms in these settings. 


\section{Preliminaries}

The stochastic process $\gamma^{t-1}$ is the key to developing the investment strategy for building the appropriate capacity in this model. It yields the posterior distribution of the unknown success rate of the Bernoulli experiments, and its terminal value $\gamma^{T}$ indicates whether or not the firm experiences demand at rate $D$ at the conclusion of the clinical trial. We consider the transition probability given in the following lemma.

Lemma 4.1 $\gamma^{t-1}$ is a Markov process and its transition probability is given as follow:

$$
\operatorname{Pr}\left(\delta^{1}=1 \mid \gamma^{0}\right)=\frac{\gamma^{0}}{\gamma^{0}+\zeta^{0}}
$$

for $t \in 2,3, \ldots, T$ :

$$
\operatorname{Pr}\left(\sum_{j=1}^{m} \delta^{t-1+j}=k \mid \gamma^{t-1}\right)=\left(\begin{array}{c}
m \\
k
\end{array}\right) \frac{\prod_{j=0}^{k-1}\left(\gamma^{t-1}+j\right) \prod_{j=0}^{m-k-1}\left(\zeta^{t-1}+j\right)}{\prod_{j=0}^{m-1}\left(\gamma^{t-1}+\zeta^{t-1}+j\right)},
$$

for $k \in\{0,1, \ldots, m\}$.

All proofs can be found in the online supplement.

The Markov property results from the assumption that the clinical experiments are independent and identical. Thus, the order in which the experiments are revealed does not matter and each result has equal weight in the posterior distribution of $p$, so $\gamma^{t-1}$ is the sufficient statistic for the upcoming transitions. Observe in equation (8) that the impact of marginal results on the transition probability decreases over time. This is not surprising - as the end of the horizon approaches, it is natural for the firm to have a better sense of whether or not the experiment will be a success. Thus, in contrast to the typical assumptions of dynamic programming models, our process $\gamma^{t}$ is time non-homogeneous, with a state space increasing in time. However, the planning horizon in our model is finite, and the overall success of the clinical trial, which in turn give raise to the demand $D$, is revealed at a predetermined period $T$. The firm's strategy in building the capacity depends heavily on its estimate of the likelihood of the event $\left\{\gamma^{T} \geq \gamma^{c}\right\}$. The following lemma is the key to our subsequent analysis:

Lemma 4.2 $\operatorname{Pr}\left(\gamma^{T} \geq \gamma^{c} \mid \gamma^{t-1}=\gamma\right)$ is an increasing function of $\gamma$.

This is intuitive for two reasons. First, more successes observed up to time $t$ implies that the firm's estimate of $p$ is higher, which implies a higher expected likelihood of more successes in the remaining test cases. Second, a large $\gamma^{t}$ means fewer successful observations are required in the 
remaining $T-t$ cases in order to meet the exogenous requirement $\gamma^{c}$. The following lemma is an immediate consequence of Lemma 4.2 .

Lemma 4.3 $\operatorname{Pr}\left(\gamma^{T} \geq \gamma^{c} \mid \gamma^{t}=\gamma+1\right) \geq \operatorname{Pr}\left(\gamma^{T} \geq \gamma^{c} \mid \gamma^{t-1}=\gamma\right)$.

In other words, a successful result improves the chance of meeting $\gamma^{c}$. Similarly, we have:

Lemma 4.4 $\operatorname{Pr}\left(\gamma^{T} \geq \gamma^{c} \mid \gamma^{t}=\gamma\right) \leq \operatorname{Pr}\left(\gamma^{T} \geq \gamma^{c} \mid \gamma^{t-1}=\gamma\right)$.

Thus, an unsuccessful trial decreases the chance of meeting $\gamma^{c}$.

\section{$5 \quad$ Investment Strategies}

Next, we characterize the optimal investment strategy in various settings. Recall that we assume it takes at least as long to complete the clinical trial as it does to build each type of production facility, so that $T \geq s_{i}^{0}$ for $i \in\{R, E\}$. Nevertheless, given the increasing quality of information, the firm should intuitively delay production as long as there is no risk of delaying production. Indeed, by employing the appropriate coupling technique, this intuition can be proven:

Lemma 5.1 Define $s_{\max }^{0}=\max \left\{s_{i}^{0} \mid i \in\{R, E\}\right\}$. Suppose $T>s_{\max }^{0}$, then

$$
V_{1}\left(\gamma^{0}, \mathbf{s}^{\mathbf{0}}\right)=\alpha^{T-s_{\max }^{0}} E\left[V_{T-s_{\max }^{0}+1}\left(\gamma^{T-s_{\max }^{0}}, \mathbf{s}^{\mathbf{0}}\right) \mid \gamma^{0}\right] .
$$

Hence, it is optimal for the firm to delay production until period $t=T-s_{\max }^{0}$.

Thus, for any starting period $t-s_{\text {max }}^{0}$, the firm can write an equivalent problem with a planning horizon of $s_{\max }^{0}$ periods by setting $\left(\gamma^{\prime 0}, \zeta^{\prime 0}\right)=\left(\gamma^{T-s_{\max }^{0}}, \zeta^{T-s_{\max }^{0}}\right)$. Hence, without loss of generality, we assume $s_{\max }^{0}=T$ in our subsequence analysis.

In the following subsections, we characterize the firm's optimal investment strategies under various settings of the dynamic program (that is, of equations (1) and (2)). We begin with a relatively straightforward setting, and then progressively consider more complicated settings.

\subsection{One Capacity Type/Stationary Cost/No Fixed Cost}

We first model the case where the firm is considering one production facility type, with per period construction cost, $c>0$. The firm can start, pause and restart construction in each of the first $T$ periods without incurring additional setup costs, so that $K=0$. If at the end of the horizon the new drug is approved but the production facility is not yet ready, the firm is then charged a fixed per-period-delayed penalty cost, $\Pi(s)=\pi s$ for each of the periods needed to complete construction. 
Note that without any setup cost, the firm's decision at period $t$ is independent of its investment history at $t-1$. In this setting, we do not have to consider the decision in the previous period, so:

$$
V_{t}\left(\gamma^{t-1}, s^{t-1}, a^{t-1}\right)=V_{t}\left(\gamma^{t-1}, s^{t-1}\right) .
$$

Thus:

$$
V_{t}\left(\gamma^{t-1}, s^{t-1}\right)=\min \left\{c+\alpha E\left[V_{t+1}\left(\gamma^{t}, s^{t-1}-1\right) \mid \gamma^{t-1}\right], \alpha E\left[V_{t+1}\left(\gamma^{t}, s^{t-1}\right) \mid \gamma^{t-1}\right]\right\}
$$

with

$$
\begin{gathered}
V_{t}\left(\gamma^{t-1}, 0\right)=0, \\
V_{T+1}\left(\gamma^{T}, s^{T}\right)= \begin{cases}s^{T} \cdot(c+\pi) & \text { if } \gamma^{T} \geq \gamma^{c} \\
0 & \text { otherwise }\end{cases}
\end{gathered}
$$

for $t=1,2, \ldots, T$. Since the penalty cost incurred at $T+1$ is linear to the number of periods that production will be delayed $s^{T}$ (or in other words, the number of periods required to complete production):

$$
\begin{aligned}
V_{t}\left(\gamma^{t-1}, s^{t-1}\right) & =\min \left\{c+\alpha E\left[V_{t+1}\left(\gamma^{t}, s^{t-1}-1\right) \mid \gamma^{t-1}\right], \alpha E\left[V_{t+1}\left(\gamma^{t}, s^{t-1}\right) \mid \gamma^{t-1}\right]\right\} \\
& =\min \left\{c, \alpha^{T-t+1}(c+\pi) \operatorname{Pr}\left(\gamma^{T} \geq \gamma^{c} \mid \gamma^{t-1}\right)\right\}+\alpha E\left[V_{t+1}\left(\gamma^{t}, s^{t-1}-1\right) \mid \gamma^{t-1}\right] .
\end{aligned}
$$

Hence, it is optimal for firm to invest in construction in period $t$ if

$$
c \leq \alpha^{T-t+1}(c+\pi) \operatorname{Pr}\left(\gamma^{T} \geq \gamma^{c} \mid \gamma^{t-1}\right) .
$$

In other words, the firm's decision involves choosing between the cost of investing at the current period $t$ and the expected marginal penalty incurred by delaying the investment.

Observation 1 Observe in (10) that the time to-build-parameter, $s^{t}$, is not a factor in the investment decision. By Lemma 5.1, we can assume without loss of generality that $s^{0}=T$, so $s^{t} \geq T-t$. Therefore, the decision is monotonic in $s^{t}$; that is, if it is optimal for the firm to invest when the time-to-build $s^{t}=s$, it is also optimal for the firm to invest at any time-to-build $s^{t} \geq s$.

Observation 2 We are able to compute the optimal investment strategy without explicitly solving the dynamic program. This is a consequence of the fact that the penalty term $s^{T}(c+\pi)$ is linear in the time-to-build term $s^{T}$.

We use inequality (10) to characterize the optimal policy in this case.

Theorem 5.2 Given a facility to build with constant per unit penalty costs and no setup cost, at each period $t \in\{1,2, \ldots, T\}$, there exists a $\gamma_{*}^{t-1} \in\left\{\gamma^{0}, \gamma^{0}+1, \ldots, \gamma^{0}+t-1\right\} \cup\{\infty\}$ such that the optimal construction strategy is given by:

$$
a_{*}^{t}= \begin{cases}1 & \text { if } \gamma^{t-1} \geq \gamma_{*}^{t-1} \\ 0 & \text { otherwise }\end{cases}
$$

where 


$$
\gamma_{*}^{t-1}:=\inf \left\{\gamma \in\left\{\gamma^{0}, \ldots, \gamma^{0}+t-1\right\} \mid \operatorname{Pr}\left(\gamma^{T} \geq \gamma^{c} \mid \gamma^{t-1}=\gamma\right) \geq \frac{c}{\alpha^{T-t+1}(c+\pi)}\right\}
$$

(Here, we use $t-1$ as the time index to match the realization of the stochastic process, $\gamma^{t-1}$ at period $t$.) This result is a direct consequence of Lemma 4.2. This threshold policy indicates that in this setting and given the problem parameters, the optimal investment strategy depends only on $t$ and $\gamma^{t-1}$, is monotonic in $\gamma^{t-1}$, and is independent of the current progress $s^{t-1}$. Because of the linearity of the penalty function, the firm can compare the construction cost and the discounted expected marginal penalty directly. Moreover, Lemma 4.2 implies that a higher $\gamma^{t-1}$ implies a higher likelihood that the new drug will be sold in the market, so that the firm is more likely to be penalized if the production capacity is not ready by time $T+1$. Thus, if it is optimal for the firm to invest if $\gamma^{t-1}=\gamma$ for some $\gamma$, it is also optimal to invest if $\gamma^{t-1}=\gamma+1$. Thus,

Corollary 5.3 Suppose $\gamma^{t-1} \geq \gamma_{*}^{t-1}$ and $\gamma^{t}=\gamma^{t-1}+1$, then $\gamma^{t} \geq \gamma_{*}^{t}$ for each $t \in\{1,2, \ldots, T\}$.

In other words, if it is optimal for the firm to invest in construction in period $t$ and the firm sees a success from the latest test result at the end of period $t$, then it is also optimal for the firm to invest in period $t+1$. Moreover:

Corollary 5.4 For $\alpha=1$ and $t \in\{2, \ldots, T\}$, if $\gamma_{*}^{t-1}<\infty$, then $\gamma_{*}^{t} \geq \gamma_{*}^{t-1}$

However, this may not be true when the discount rate $\alpha$ is less than 1 . Consider the following counterexample:

Example 1 Consider the setting in which the firm has preliminary data $\gamma^{0}=\zeta^{0}=5$, the adjusted threshold, $\gamma^{c}=24$, there are 48 periods in the planning horizon, the per period building cost $c=\$ 10$ Million, the per period penalty cost $\pi=\$ 100$ Million, and the per period discount rate $\alpha=0.9$. In this case, the threshold is decreasing in periods 26,27 and 28 (see Figure 1).

There is no monotonicity in this case because the firm is comparing the investment cost and the discounted expected marginal penalty, and for a long enough planning horizon, the discount factor, which is exponential in time, makes the investment in the current period less attractive than in subsequent ones.

\subsection{Positive Setup Cost, $K>0$}

It can often be expensive to start or restart a construction project due to hiring, resource procurement, etc. To explore the implications of these additional costs, we extend the model from the previous subsection by adding a positive setup cost $K>0$ that is incurred when a project is started or restarted after construction has not taken place in the previous period. 


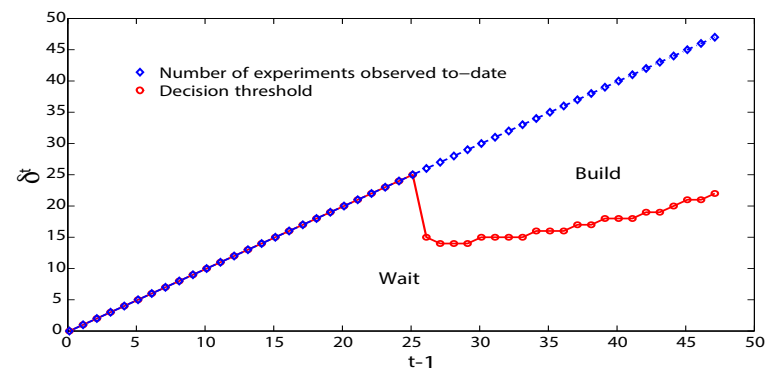

Figure 1: Non-monotonic investment threshold with $\alpha<0$.

In this setting, the optimal investment strategy in period $t$ is characterized by a pair of thresholds depending on whether or not the construction project was underway in the previous period:

Theorem 5.5 In the setting described above, with linear terminal penalty function and a single capacity type with positive setup cost $K>0$ charged when construction is started or restarted after an idle period, for each $t \in\{1, \ldots, T\}$ there exists a pair of threshold values $\gamma_{*_{0}}^{t-1}, \gamma_{*_{1}}^{t-1} \in$ $\left\{\gamma^{0}, \gamma^{0}+1, \ldots, \gamma^{0}+t-1\right\} \cup\{\infty\}:$

$$
\begin{gathered}
\gamma_{*_{0}}^{t-1}:=\inf \left\{\gamma \in \Gamma \mid \operatorname{Pr}\left(\gamma^{T} \geq \gamma^{c} \mid \gamma^{t-1}=\gamma\right) \geq \frac{c+K+\alpha E\left[W_{t+1}\left(\gamma^{t}, s^{t-1}-1\right) \mid \gamma^{t-1}\right]}{\alpha^{T-t+1}(c+\pi)}\right\}, \\
\gamma_{*_{1}}^{t-1}:=\inf \left\{\gamma \in \Gamma \mid \operatorname{Pr}\left(\gamma^{T} \geq \gamma^{c} \mid \gamma^{t-1}=\gamma\right) \geq \frac{c+\alpha E\left[W_{t+1}\left(\gamma^{t}, s^{t-1}-1\right) \mid \gamma^{t-1}\right]}{\alpha^{T-t+1}(c+\pi)}\right\},
\end{gathered}
$$

where

$$
W_{t+1}(\gamma, s):=V_{t+1}(\gamma, s, 1)-V_{t+1}(\gamma, s, 0), \quad \Gamma=\left\{\gamma^{0}, \ldots, \gamma^{0}+t-1\right\}
$$

such that

- $\gamma_{*_{0}}^{t-1} \geq \gamma_{*_{1}}^{t-1}$

- If $a^{t-1}=0, a_{*}^{t}=1$ if $\gamma^{t-1} \geq \gamma_{*_{0}}^{t-1}$ otherwise $a_{*}^{t}=0$, and

- If $a^{t-1}=1, a_{*}^{t}=1$ if $\gamma^{t-1} \geq \gamma_{* 1}^{t-1}$ otherwise $a_{*}^{t}=0$.

In other words, there are two thresholds $\gamma_{*_{0}}^{t-1}$ and $\gamma_{*_{1}}^{t-1}$ governing the firm's investment strategy, depending on the action in the previous period. If the firm invested in the construction project in the previous period so that $a^{t-1}=1$, then the firm should invest in period $t$ if and only if $\gamma^{t-1} \geq \gamma_{*_{1}}^{t-1}$. Likewise, if the firm did not invest in the previous period, $a^{t-1}=0$, then the firm should invest in period $t$ if and only if $\gamma^{t-1} \geq \gamma_{*_{0}}^{t-1}$.

The relationship between the two thresholds $\gamma_{*_{0}}^{t-1} \geq \gamma_{*_{1}}^{t-1}$ reflects the fact that the setup cost introduces an entrance fee to the investment, and thus the firm needs to have more confidence in the event $\gamma^{T} \geq \gamma^{c}$ before investing if it did not in the previous period. To compare the solution to the no setup cost case from Subsection 5.1, we observe the following:

Corollary 5.6 For the optimal thresholds $\left\{\gamma_{*_{1}}^{t-1}, \gamma_{*_{0}}^{t-1}\right\}$ at period $t$ for $K>0$, and given the same set of parameters as the model characterized in Theorem 5.2 with an optimal threshold in period $t$ 
of $\gamma_{*}^{t-1}$, then

$$
\gamma_{*_{1}}^{t-1} \leq \gamma_{*}^{t-1} \leq \gamma_{*_{0}}^{t-1}
$$

In other words, the decision threshold level from the no setup cost model is sandwiched between the pair of threshold levels for the model with setup cost.

\subsection{Increasing Marginal Penalty Cost}

Up to now, we have implicitly assumed the penalty cost function models lost sales during the time periods that the commercial production of the new drug is delayed due to lack of production capacity. We therefore modeled it as a constant per unit cost, $\Pi(s)=\pi s$. In some cases, however, we need to account for loss of goodwill, patent life, patients' health, etc. In general, we can consider a penalty cost function $F\left(s^{T}=s, a^{T}=a\right)$, where the amount penalized is a function of the number of periods $s$ production is delayed. Indeed, in many cases, the longer the production delay, the more damage the firm suffers, so that the marginal penalty cost is an increasing function of the number of periods $s$ required to complete construction after time $T$, and thus:

$$
F(s+1, a)-F(s, a) \geq F(s, a)-F(s-1, a) .
$$

Note that we are considering a discrete time model so $s \geq 0$ takes integer values. Inequality (12) implies that $F(s, a)$ is a convex function on the discrete set $s$.

Theorem 5.7 In the setting described above with a non-negative, increasing, convex penalty function satisfying condition (12), zero setup cost, and a single type of construction project, for each $s \geq T-t+1$ and $t \in\{1,2, \ldots, T\}$, there exists a threshold value $\gamma_{*}^{t-1}(s) \in\left\{\gamma^{0}, \gamma^{0}+1, \ldots, \gamma^{0}+t-\right.$ $1\} \cup\{\infty\}$, such that:

Moreover,

$$
a_{*}^{t}(s)= \begin{cases}1 & \text { if } \gamma^{t-1} \geq \gamma_{*}^{t-1}(s), \\ 0 & \text { otherwise. }\end{cases}
$$

- $\gamma_{*}^{t-1}(s) \geq \gamma_{*}^{t-1}(s+1)$,

- $V_{t}\left(\gamma^{t-1}, s\right)-V_{t}\left(\gamma^{t-1}, s-1\right) \geq 0$ and increases in $\gamma^{t-1}$, and

- $V_{t}\left(\gamma^{t-1}, s\right) \geq 0$ is convex in $s$ for all $t \in\{1,2, \ldots, T\}$.

Observe that $\gamma_{*}^{t-1}(s)$ is decreasing in $s$, which is due to the increasing marginal penalty cost. This suggests that when there is still much to be built and the end of the horizon is approaching, the firm employs an increasingly aggressive investment strategy in order to reduce the duration of the potential delay in production. The expected total cost in an increasing function of $s$ and the differences increases in $\gamma^{t-1}$ because the expected penalty cost increases in both the remaining time-to-build and the probability of a successful trial. 


\subsection{Two Investment Projects}

Up to this point, our models have reflected one key trade-off faced by the firm - the trade-off between investing early on to ensure that sufficient production capacity will be available at product launch, and waiting until additional information is collected to avoid unnecessary investment. Increasingly, however, firms are considering alternative types of production capacity that are faster to build, and thus allow firms to delay capacity projects without impacting the availability of the product if it is approved. As discussed in more detail in the Introduction, biopharmaceutical firms are beginning to explore the use of emerging alternative manufacturing approaches built around smaller-scale disposable technology, such as disposable tanks and mixers that are pre-sterilized, pre-validated and self-contained, significantly reducing capacity acquisition lead time. Clearly, if this capacity proves to be less expensive than traditional capacity to build and operate, it dominates the traditional capacity. However, it remains unclear at this time whether or not this capacity will be less expensive to install, and it is very likely that this capacity will be more expensive to operate in the long run. Recall that we model this in (4)-(6), with two capacity project types regular (Project R) and expedited (Project E). Recall that the constant $O$ in the terminal value function

$$
F\left(\mathbf{s}^{\mathbf{T}}\right)=\min \left\{s_{R}^{T} \cdot\left(\pi+c_{R}\right), s_{E}^{T} \cdot\left(\pi+c_{E}\right)+O\right\} .
$$

allows us to model the potentially higher operating cost of Project E.

From Lemma 5.1, we know that the firm will not consider investing in Project E until period $t=T-s_{E}^{0}$. The problem can be reduced to a two-stage problem, where at each period in Stage $1, t \in\left\{1, \ldots, T-s_{E}^{0}-1\right\}$, it is feasible to either investment in Project $\mathrm{R}$, or to not invest, while for each period in Stage 2, $t \in\left\{T-s_{E}^{0}, \ldots, T\right\}$, it is feasible to invest in either project, or neither project. Although the firm will not invest in Project E until the start of Stage 2, the availability of this more expensive alternative may impact the firm's decision making in Stage 1. To partially characterize the optimal strategy, we first address the Stage 2 problem.

\subsubsection{The Stage 2 Problem}

For each period in the Stage 2 problem, we partially characterize the optimal policy as follows:

Lemma 5.8 For each period $t$ in Stage 2, for any $s^{t-1}$, there exist two values $S_{u}^{t-1}, S_{d}^{t-1}$ :

$$
S_{u}^{t-1}:=\frac{s^{t-1}\left(\pi+c_{R}\right)-O}{\pi+c_{E}}+k_{1}^{t}
$$


and

$$
S_{d}^{t-1}:=\frac{s^{t-1}\left(\pi+c_{R}\right)-O}{\pi+c_{E}}+k_{2}^{t},
$$

where

$$
k_{1}^{t}:=\frac{c_{R}}{c_{E}}+(t) \frac{c_{E}-c_{R}}{\pi+c_{E}},
$$

and

$$
k_{2}^{t}:=\frac{c_{R}}{c_{E}}-(t+1) \frac{\pi+c_{R}}{\pi+c_{E}} .
$$

such that if $s_{E}^{t-1} \geq S_{u}^{t-1}$,

and if $s_{E}^{t-1}<S_{d}^{t-1}$,

$$
V_{t}\left(\gamma^{t-1}, s_{R}^{t-1}, s_{E}^{t-1}\right)=V_{t}\left(\gamma^{t-1}, s_{R}^{t-1}\right)
$$

$$
V_{t}\left(\gamma^{t-1}, s_{R}^{t-1}, s_{E}^{t-1}\right)=V_{t}\left(\gamma^{t-1}, s_{E}^{t-1}\right) .
$$

In other words, for each period $t$ in Stage 2 , we can identify regions in the $\left(s_{R}^{t-1}, s_{E}^{t-1}\right)$-space where we can reduce the 2 Project model to an equivalent 1 Project model. At each period $t$ in Stage 2 , these regions are defined by two parallel lines with slope $\left(\pi+c_{R}\right) /\left(\pi+c_{E}\right)$ as described in Lemma 5.8. Above the upper boundary (14), the firm will not consider building Project E; below the lower boundary (15), the firm will not consider building Project R. In the intermediate region, both projects must be considered.

Clearly, the firm's decision depends on the remaining time to complete each of the projects, and the larger the difference, the clearer the decision. If the firm has been progressing on Project R, it may be able to drop Project E from consideration relatively early. For example, if the clinical trial has shown positive results since start, and the firm has been consistently building Project R, by the time that Project E should be considered, the two projects may result in the same number of periods to work on until finish. In this case, the firm should obviously commit to the cheaper Project R. Conversely, if the firm has been delaying construction of Project R, it may reach the point relatively early where the penalty cost from commiting to Project $\mathrm{R}$ due to the long build time exceeds the savings in construction cost. Thus, there is no value in considering Project R.Figure 2 illustrates the "gray area" between the boundaries (14) and (15) where both of the projects are still under consideration. Although this "gray area" expands as we move further from the terminal period, in our computational experiments (in the next section) the firm rarely switches project in Stage 2. Intuitively, this "gray area" is the region that comes into play if the current data suggests the trial is on the borderline between passing and not passing, but in most of our experiments it was extremely rare for the problem to remain within this region for an extended period of time. 

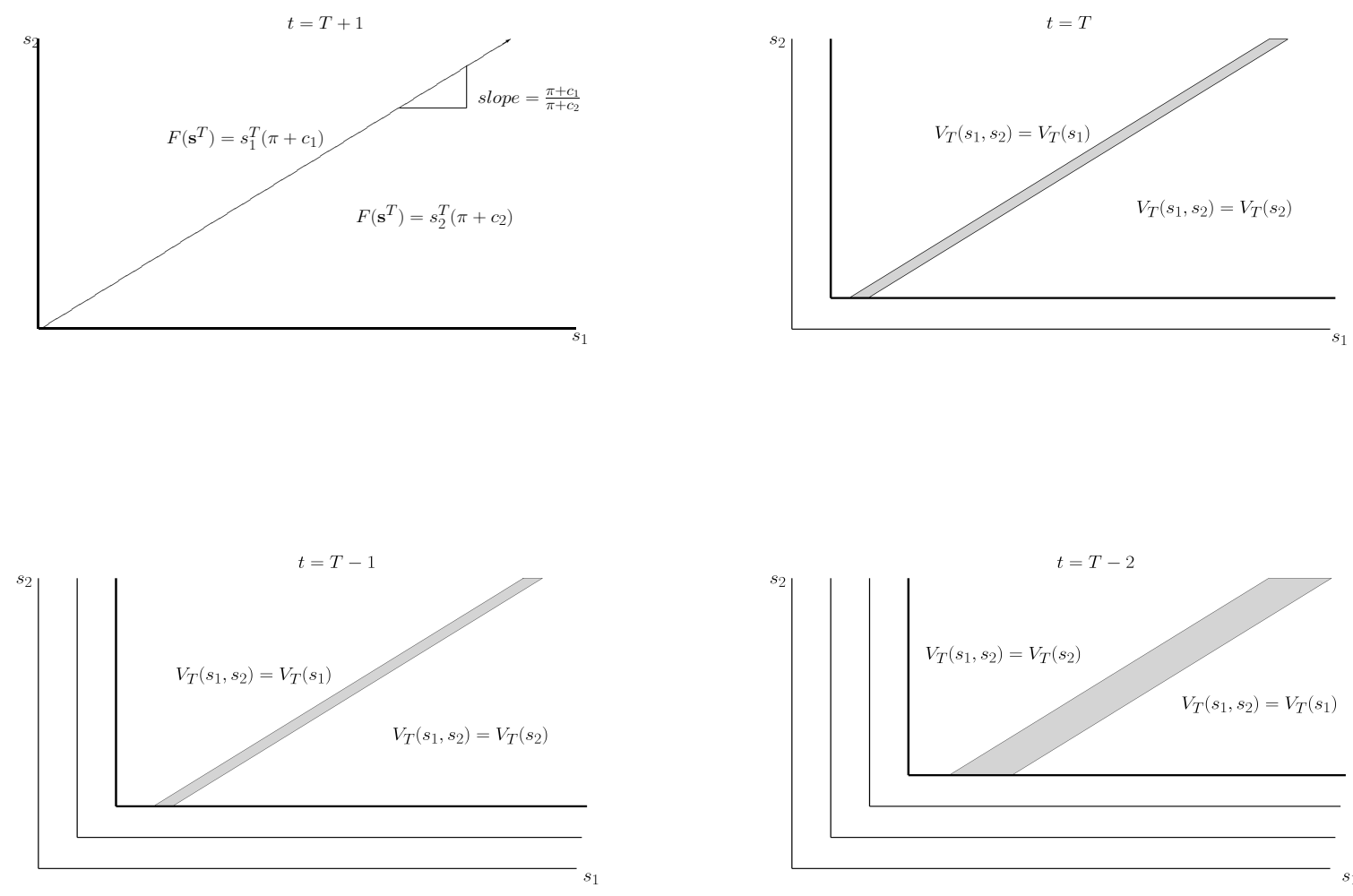

Figure 2: The pair of decision boundaries where the 2 project problems can be reduced to a single project problem.

\subsubsection{The Stage 1 Problem}

In Stage 1, the firm decides whether or not to build Project $\mathrm{R}$ in each period. The corresponding dynamic program for periods $t \in\left\{1, \ldots, T-s_{E}^{0}-1\right\}$ can be written as:

$$
V_{t}\left(\gamma^{t-1}, s_{R}^{t-1}, s_{E}^{0}\right)=\min _{a_{R}^{t} \in\{0,1\}}\left\{a_{R}^{t} c_{R}+\alpha E\left[V_{t+1}\left(\gamma^{t-1}+\delta^{t}, s_{R}^{t-1}-a_{R}^{t}, s_{E}^{0}\right) \mid \gamma^{t-1}\right]\right\},
$$

with terminal value function,

$$
V_{T-s_{E}^{0}}\left(\gamma^{T-s_{E}^{0}-1}, s_{R}^{T-s_{E}^{0}-1}, s_{E}^{0}\right)
$$

Although this dynamic programming formulation resembles the problem in Section 5.1, it turns out that in general the optimal building decision of the dynamic program $(16,17)$ is dependent on the time-to-build $s_{R}^{t-1}$ and is not monotonic in $s_{R}^{t-1}$ as we illustrate in Example 2.

Example 2 Consider an example with preliminary data $\gamma^{0}=\zeta^{0}=5$, adjusted threshold $\gamma^{c}=24$, 48 periods in the planning horizon, per period building costs for Project $\mathrm{R}$ and Project $\mathrm{E}$ of $c_{R}=\$ 10$ million and $c_{E}=\$ 20$ million respectively, per period penalty cost of $\pi=\$ 100$ million, per period 
discount rate of $\alpha=0.99$, and Project E lead time of 36 periods. At period $t=11$, with $s_{R}^{10}=41$ the firm should build at period $t$ if $\gamma^{10} \geq 3$ and with $s_{R}^{10}=42$ the firm should only build when $3 \leq \gamma^{10} \leq 7$

In this case, if the firm does not build for more than 4 periods by $t=11$ and $\gamma^{10} \geq 7$ implying a good chance of passing the trial, the potential penalty of delaying for more than 4 periods is too high, so the firm will abandon Project R, and focus on Project E in Stage 2 instead. However, if the firm misses only 3 periods by period $t=11$, the savings from building the more economical option, Project R, may compensate for the lost in delaying production, and the firm will build Project $\mathrm{R}$ for period $t=11$.

\subsection{Discussion}

Of course, the models in this section are highly stylized, significantly simplifying both capacity acquisition and clinical trial data collection. Nevertheless, the results serve to both give insights into effective managerial practices, and to facilitate the computational analysis discussed in the next section of this paper. The core takeaway, that it can be valuable to reassess investment decisions periodically, is supported both by our analytical results, and the computation below. We believe that other insights, such as the impact of the cost of restarting paused projects on ongoing investment decision-making, the increased aggressiveness at the end of the horizon resulting from increasing marginal penalty costs, and the notion of "regions" where one of two competing projects can be excluded from consideration, are likely to conceptually hold in more complex settings.

\section{Computational Study}

In the previous sections of this paper, we developed a stylized model to characterize decision-making about capacity investment decisions as information is updated during a clinical trial. Building on our characterization of optimal facility construction strategies, we present the results of a series of computational experiments we complete to give insight into the following questions:

- How does an approach in which capacity investment decisions are regularly re-evaluated as available information changes compare to more traditional approaches? How often should investment decisions be reconsidered?

- In this paper, we propose an approach that explicitly accounts for the fact that information becomes more certain as time and trials progress. How important is it to account for this 
improving quality of information over time?

- How valuable is the option to stop and restart projects? How does this compare to a setting in which the firm can consider investing regularly, but must complete the construction of a project once investment has started?

- What is the impact of setup costs and increasing marginal penalty costs on total costs and on decision-making?

- If a firm has the option of investing in an alternative production capacity that is more expensive but has a shorter capacity lead time, should it? Does it ever make sense to invest in both traditional capacity and expedited capacity during the trial period?

We explore these questions under a variety of problem parameters such as costs, data quality, and horizon length, to explore how the problem setting impacts the answer to these questions.

\subsection{Experimental Design}

To address the questions raised above, for a variety of parameter settings we compare a variety of alternative strategies (depending on the particular question we are addressing) for making capacity investment decisions in addition to the optimal strategy characterized in this paper. In each case, we first solve for the investment strategy, then compute the performance measures via simulation.

As our stylized model is not intended to capture many real-world complications of clinical trials and capacity investment, our goal in these experiments is to develop insight into the potential impact of the updating process on the firm's decision-making. Our experimental parameters are thus intended to capture a broad range of potential situations faced by firms when building production capacity in this setting. In practice, even in settings very similar to those captured in our models, precise estimates of each of these parameters will be difficult to make. In particular, while capacity construction costs can be estimated, reliably estimating fixed costs associated with restarting projects, and penalty costs associated with not completing projects on time, is quite challenging. Nevertheless, our computational experiments give insight into the qualitative impact of relatively high or relatively low penalty or fixed costs, regardless of precise values.

We consider the case without setup cost $(K=0)$ and the following set of parameters: We fix the problem horizon to $T=\mathbf{2 5 0}$ weeks (periods), or five years at 50 weeks per year. This is slightly less than the time it will take for a new drug to go through the three stages of clinical trials, and roughly the time it will take to build and qualify new capacity. We use a discount rate of $\alpha=\mathbf{0 . 9 9 9}$ per week, corresponding to an annual discount rate of 0.95 . The new drug is approved 
if the number of successful clinical cases exceeds $\gamma^{c}-\gamma^{0}=\mathbf{2 0 0}$. To assess the sensitivity of our results to prior information quality, we vary $n_{0}=\{0,5, \mathbf{1 0}\}$. We vary per period investment costs as follows: $c_{R}=\{\$ 1, \$ \mathbf{2}, \$ 4\}$ million per week for 250 weeks required to complete the traditional capacity. Upon approval of the new drug, if capacity is not yet completed we consider penalty costs that vary as follows: $\pi=\{\$ 10, \$ \mathbf{2 0}, \$ 50\}$ million for each week of delay in addition to the investment necessary to complete the project. For each set of experimental parameters, we compute the optimal decisions in all relevant states (as well as the decisions using other policies described in the sections below), simulate 2,500 sample paths of $\gamma^{t}$, and calculate our performance measures for each of our policies. For each of these simulation runs, we randomly generate the true success rate, $p$, from a uniform $(0,1)$ distribution. To simulate the prior information, we generate $\gamma^{0}$ and $\zeta^{0}$ from a Bernoulli distribution using parameters $p$ and $n_{0}$. In what follows, when we refer to a base case we are referring to those parameters that are boldfaced above.

In the following subsections, we address the questions raised above by comparing our optimal approach to alternative approaches intended to model other practices. For each of our experiments, we consider three performance measures: the expected value of total cost over the horizon, which includes building cost (regardless of whether or not the product is approved) and penalty cost if capacity is not ready in time; conditional expected penalty cost if the product is approved; and conditional expected building cost if the product is not approved. These last two measures in some sense capture the cost of wrong decisions.

\subsection{Frequency of Reconsideration}

In this subsection, we address two related questions: when does the approach we outline in this paper make sense, and if this approach is implemented, how frequently does it make sense to reconsider investment.

Recall that in our model, preliminary data, prior lab results, and expert opinions are collectively modeled by $\left(\gamma^{0}, \zeta^{0}\right)$. To address the first question, we compare our optimal approach to one in which the firm simply uses this distribution of $p, \operatorname{Beta}\left(\gamma^{0}, \zeta^{0}\right)$, to calculate the probability of a successful trial $\gamma^{T} \geq \gamma_{c}$ at the terminal period $T+1$, in order to decide whether or not to commit to building the production capacity. If $E$ [Total Cost $\mid$ Success $] \operatorname{Pr}($ Success $) \geq E[$ Total Cost|Failure $] \operatorname{Pr}($ Failure), the firm will start the construction. Otherwise, the firm will not build the capacity - we call this the Traditional approach. (Note that since we know $\gamma^{0}$ and $\gamma^{c}$, we can calculate $\operatorname{Pr}$ (Success) and $\operatorname{Pr}($ Failure) using equation (8).) Once the decision is made, the firm does not change its strategy 
throughout the planning horizon. This is intended to model the typical practice of making the decision whether or not to invest in capacity in time to be able to complete the capacity before product approval, and to not reconsider that decision after it is made. To explore the effect of the frequency of reconsidering investment, we compare our optimal approach to one in which estimates are updated and investments are reconsidered at the end of every $m$ periods, where $m=25$ or $m=50$. The results of our experiments comparing the optimal approach, the Traditional approach, and these intermediate approaches with less frequent updating, appear in Figures 3(a) (the overall expected cost), 3(b) (the expected penalty cost given success) and 3(c) (the expected "wasted" investment given failure).

(a) Expected Total Cost

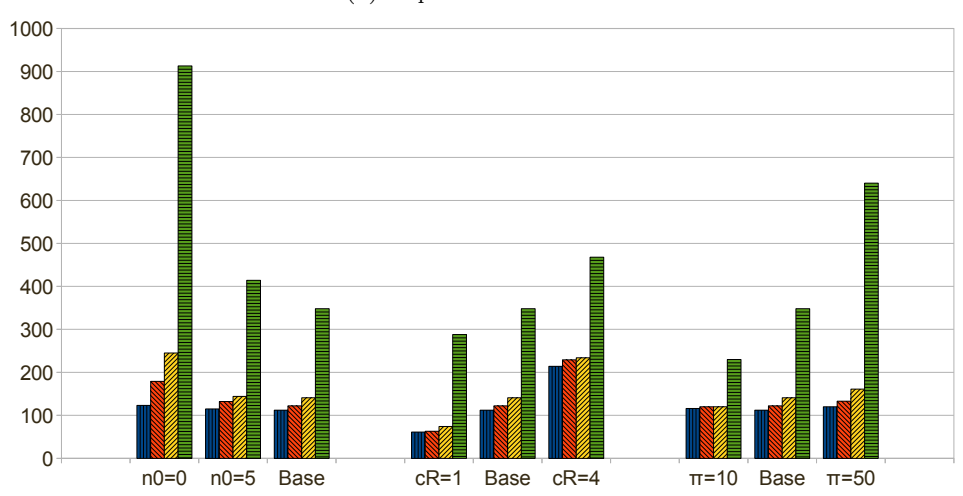

(b) Expected Penalty Given Success

(c) Expected Investment Cost Given Failure
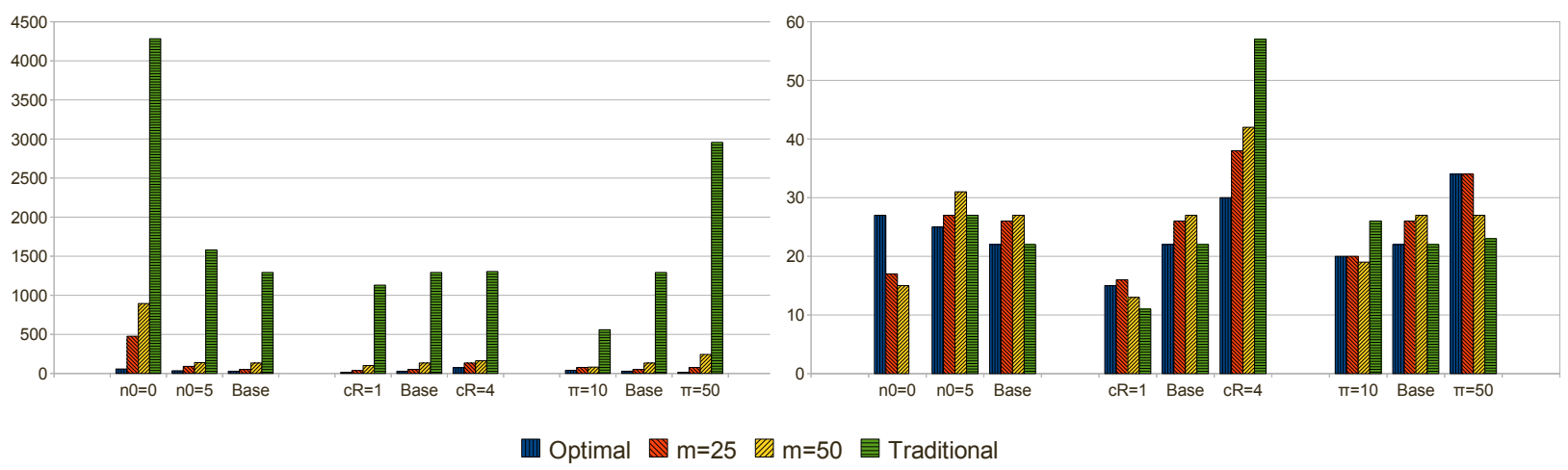

Figure 3: Expected total costs with various updating frequencies in $\$$ million.

Our experiments indicate that the information updating process can significantly reduce the expected cost of building capacity for a new drug. In our experiments, we see that on average, following our optimal approach leads to an $70 \%$ decrease in expected cost. In Figure 3(a), we see that this gain is particularly dramatic when little initial information is available, or penalty costs are particularly high. In Figure 3(a), we see similarly dramatic gains in controlling penalty costs if the trial is successful (again, particularly with poor initial information or high penalty costs). However, when the drug fails the approval process, the traditional approach often dominates our 
approach in terms of "wasted" investment dollars, as illustrated in Figure 3(b) (although even the biggest of these wasted investments is significantly smaller than the penalty cost if the drug is a success). This suggests a reluctance to invest in the traditional approach.

We also see that the gains from more frequently reviewing data and updating estimates are significantly smaller than the gain from moving from the traditional approach to an updating approach, even with less frequent updates. If initial information is scarce, we do see gains of $50 \%$ as we move from annual to weekly updates, and on average we see gains of $21 \%$.

Interestingly, for our optimal strategy, expected total costs increase sublinearly in both capacity and penalty costs - this is particularly dramatic in the case of penalty costs, where there is little increase in total expected cost with a five times increase in penalty costs. This suggests that as penalty costs increase, regular adjustment of the investment strategy and careful balancing of potential penalty costs and investment losses will lead to the greatest gain.

\subsection{Improving Information}

Recall that our optimal approach explicitly accounts for the fact that information becomes more certain as time and trials progress. We explore how important this is by comparing our optimal approach to one in which the firm regularly reconsiders its investment decision, but gauges the probability of the ultimate trial success solely on how many successes it has already seen by period $t$ without explicitly updating the Bayesian estimate $\hat{p}$. Thus, the firm uses the initial probability estimate $\hat{p_{0}}$ over the entire planning horizon to determine whether or not to make investments.

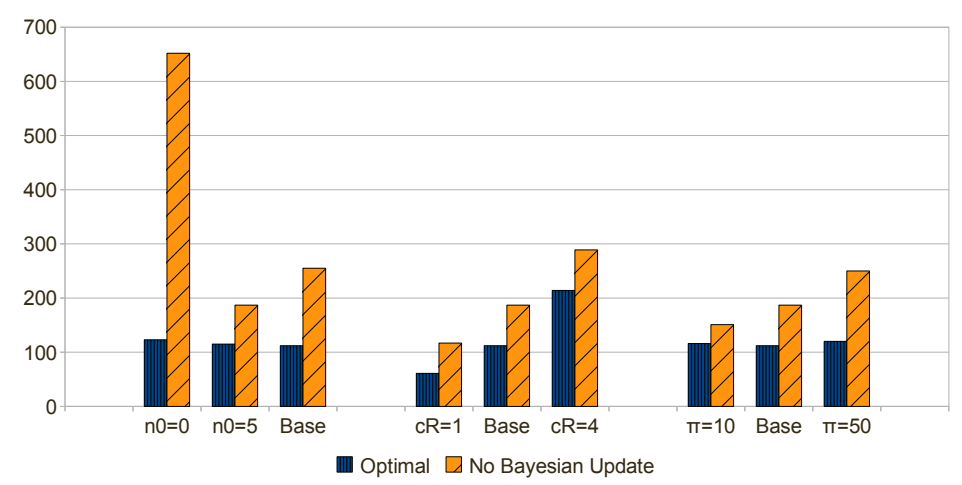

Figure 4: Expected total cost with and without Bayesian updates, in $\$$ million.

In Figure 4, we see that without explicitly updating the Bayesian estimate $\hat{p}$ the construction project costs significantly more on average, particularly when initial information is not available or penalty costs are high. If it does not update its estimate of $\hat{p}_{t}$, the firm is not using all of its available information to calculate the likelihood that the drug is approved. In particular, with 
positive intermediate results, updating the Bayesian estimate will increase $\hat{p}_{t}$, so not updating leads the firm to underestimate the true value of $p$. As a result, the firm on average will take longer to invest in capacity, resulting in the potential for more penalty charges. Indeed, we observed that on average the expected penalty cost given success was $94 \%$ higher when Bayesian updating was not used, but the expected wasted investment cost given failure was $47 \%$ higher when Bayesian updating was used.

\subsection{Pausing and Restarting Projects}

The models in the paper have two key advantages over existing practice: Bayesian updating of the estimate of final approval, and the opportunity to stop and restart capacity investment as information is updated. To isolate the benefit of stopping and restarting investment, we compared our optimal approach to one in which the firm can consider investing in each period, but must complete construction of the project once the investment has started. Although space limitations preclude presentation of detailed results, we found that expected total cost was on average $20 \%$ higher if stopping and restarting was not allowed. This was most dramatic for the lowest capacity cost and highest penalty cost experiments, where costs were $26.5 \%$ and $27.2 \%$ higher, respectively, if stopping and restarting was not allowed.

Overall, the firm stops and restarts the project in $17 \%$ of our base case parameter experiments. $12 \%$ of this $17 \%$ led to a successful launch, while the rest corresponded to negative trial results. Given that the firm stops and restarts a project, the number of restarts ranges from 1 to 12 times, and averages 3.1 times. Thus, stopping and restarting is used less than a fifth of the time, but when stopping and restarting is not allowed, the expected total cost goes up $20 \%-30 \%$. This is due to the nature of the Bayesian estimations. If initial data is such that the initial Bayesian estimate is far off, the initial investment decisions can ultimately prove to be wrong. Of course, the Bayesian estimate will effectively correct itself with later updates, but if the firm doesn't have the opportunity to update its decision, it is more likely to either build unnecessary capacity or face penalty charges. That may explain why the expected total cost is higher if we don't allow switching in the relatively small number of examples for which it is preferred.

\subsection{Positive Setup Cost, $K>0$}

As discussed in Section 5.2, it can often be expensive to start or restart a construction project due to hiring, resource procurement, etc. In that section, we explored the implications of these 
additional costs and the changes on the optimal decisions. The setup cost $K$ creates "resistance" for the decision maker to switch between the build and pause regimes. In this section, we further explore the impact of setup costs. We set all modeling parameters to base case values and vary setup cost, so that $K=0,1,2,5,10,20,50 \$$ million (or in other words, from .5 to 25 times the per period investment cost). This broad range is intended to model the spectrum of potential setup/restarting costs, from having some extra materials delivered to job site at one extreme, to having to rebid a job and rehire an entire construction crew at the other. We then measure the changes in the expected number of switches from one decision regime to another over the entire decision horizon, as well as the expected total cost, the expected investment cost given failure, and the expected penalty cost given success and summarize these results in Figures 5, 6, 7, and 8.

\section{Average Switches}

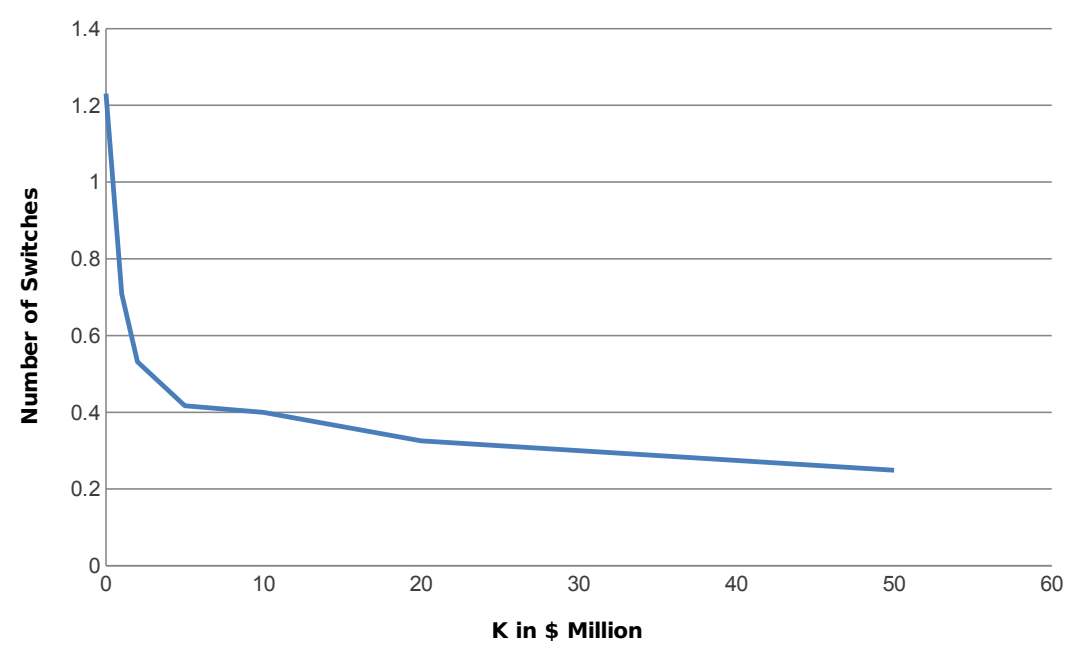

Figure 5: Average Switches vs $K$.

The number of switches decreases as $K$ increases since the decision maker tries to avoid paying setup cost. Recall from Section 5.2 that the setup cost creates a "resistance gap" between the building and not building levels in the base $K=0$ solution. This "resistance gap" encourages the decision maker to maintain the current investment state for "borderline" cases where switching should have occurred in the base case. The number of switches drops most dramatically between $K=0$ and $K=1$. Even a small positive setup cost is sufficient to create this "resistance gap", and further increases in setup cost do not have much additional impact. 


\section{Expected Total Cost}

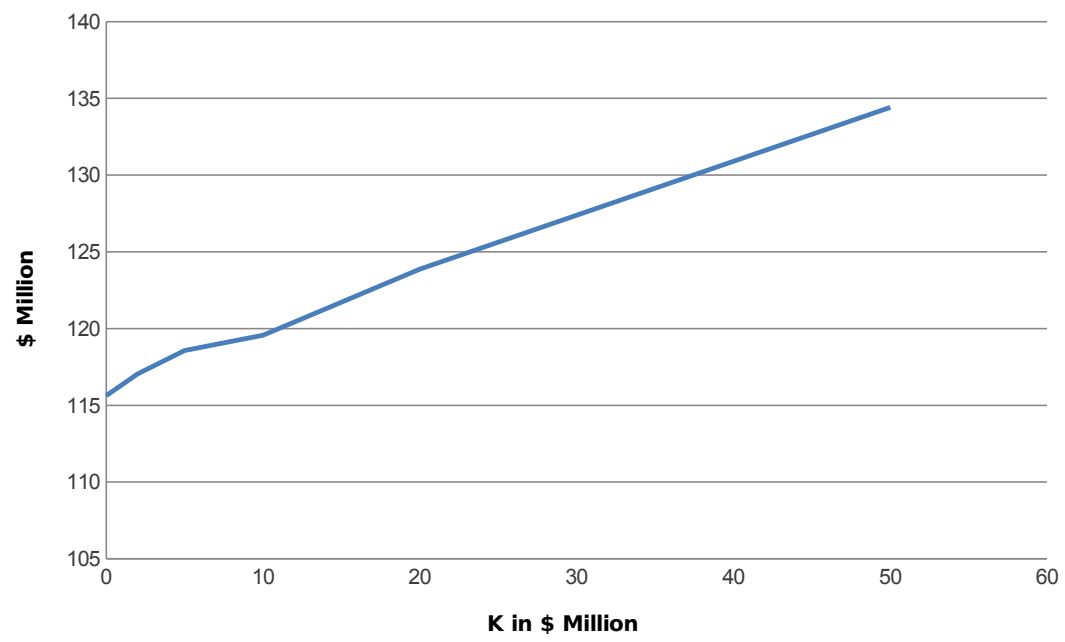

Figure 6: Expected Total Cost vs $K$.

\section{Expected Investment Cost Given Failure}

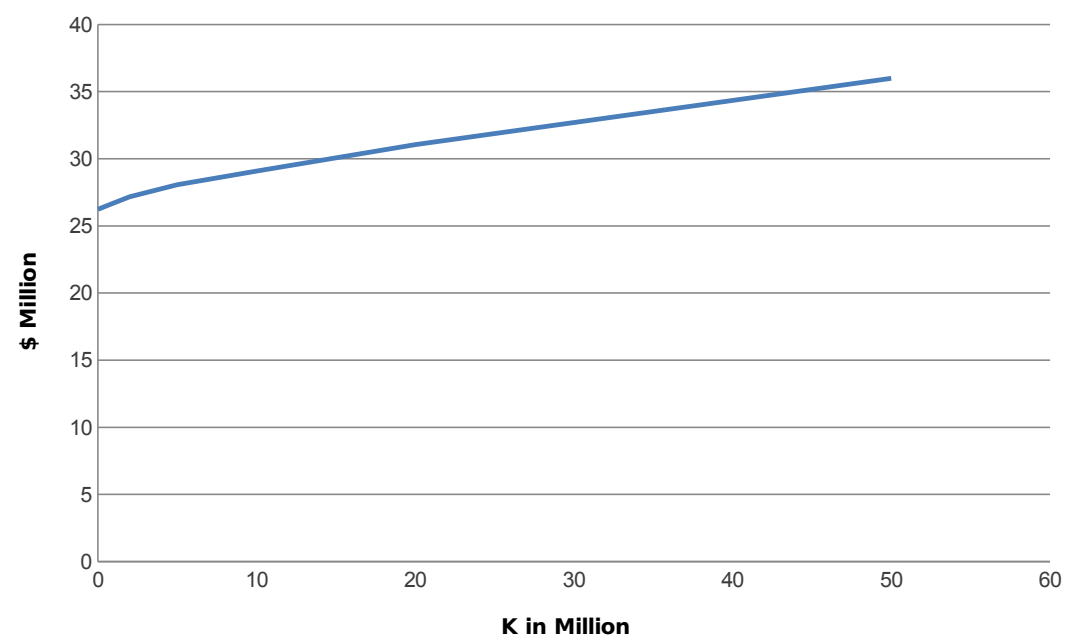

Figure 7: Expected Investment Cost Given Failure vs $K$. 


\section{Expected Penalty Cost Given Success}

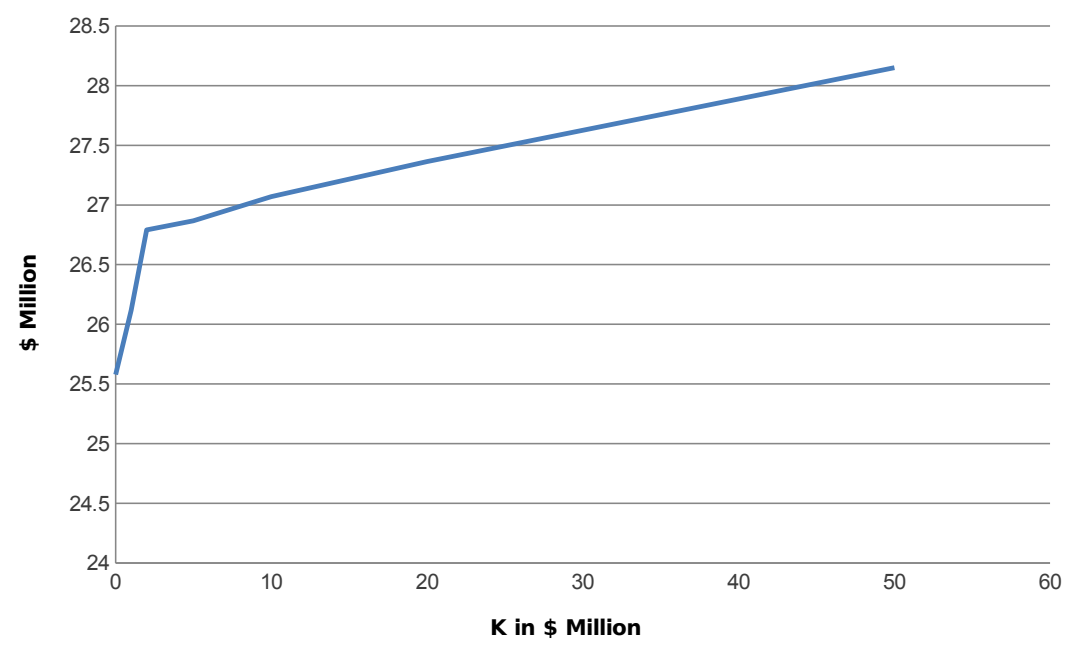

Figure 8: Expected Penalty Cost Given Success vs $K$.

As expected, all costs increase as $K$ increases. The increase in total cost is partially due to the setup cost itself, and partially due to the "resistance gap" that discourages changing investment policies. In other words, in order to avoid the cost of changing investment decisions, the decision maker has to take more risks, as illustrated in Figures 7 and 8.

These effects are not unique to the base case setting. In additional testing, we observed that even small positive setup costs have a similar effect when other costs change, and in the increasing marginal penalty cost and two projects settings.

\subsection{Increasing Marginal Penalty Cost}

In Section 5.3, we discuss why marginal penalty cost will often be increasing in amount of delay due to the loss of goodwill, patent life, patients' health, etc. This non-linearity, however, effectively reduces our ability to decrease the dimensionality of the problem, making the analysis of the solution quite complex. In this section, we computationally study the implications of increasing marginal penalty cost.

We need $f(s)$ to be a super-linear function in order to model increasing marginal penalty cost. The Exponential function is a natural extension of our original linear model. In this experiment, 
we choose a power of 1.2 for every period $s$ that the launch is delayed. Therefore, the penalty function takes the form

$$
f(s)=6.6289(s)^{1.2}
$$

We select 6.6289 to be the multiplicative constant for this penalty function so that in the "worst case scenario" $s=T$, the total penalty cost is close to that in our original linear model. This gives us a total discounted construction cost comparable to the base linear penalty case. For comparison purposes, we keep all other modeling parameters the same as the base case, and compute the expected number of switches and the expected costs as before. The results are summarized in Table 1.

\begin{tabular}{|l|c|c|c|}
\hline & $E[$ Penalty|Success] & $E[$ Investment|Failure $]$ & $E[$ Total Cost $]$ \\
\hline Increasing Marginal Penalty & $\$ 32.6$ million & $\$ 20.4$ million & $\$ 113.1$ million \\
Linear Penalty & $\$ 25.6$ million & $\$ 26.2$ million & $\$ 115.6$ million \\
\hline
\end{tabular}

Table 1: Increasing Marginal Penalty Cost vs. Linear Penalty Cost

In the increasing marginal case, we observe a higher expected penalty cost given success, a lower expected investment cost given failure, and a slightly lower expected total cost. This is because when $s$ is small, the marginal penalty cost is lower than that of the linear case. That means that the lower marginal cost at the beginning of the planning horizon encourages the decision maker to wait for more information to arrive before committing to construction, explaining the results we see for investment and penalty components of the total cost. Since the decision maker has the luxury of waiting to start the construction project, the decision maker also sees a lower expected total cost in the increasing marginal case. For this reason, we can expect a larger difference between the exponential penalty model and the base case linear version when a higher exponential power is in used.

\subsection{Two Projects}

Finally, we consider a setting in which the firm has two production capacity types to choose from, regular (Project R) and expedited (Project E). Project $\mathrm{R}$ has a longer lead time than Project E, but lower total costs. For our computational experiments in this subsection, we utilize a planning horizon of 100 periods, and a setting in which the drug is approved if the number of successful individual trials exceeds $\gamma^{c}-\gamma^{0}=80$. Project $\mathrm{R}$ has a per period investment cost $c_{R}=\$ 2$ million, and requires 100 periods to builds. Project E construction costs and construction times varied as follows: $c_{E}=\$ 4$ million with 40 weeks construction time, $c_{E}=\$ 8$ million with 30 weeks 
construction time, and $c_{E}=\$ 16$ million with 20 weeks construction time. Also, as we discussed in Section 3, to model the potentially higher operating cost of expedited capacity, the firm is charged the discounted "rolled-up" operating cost $O=\{\$ 0, \$ 50, \$ 100\}$ million at $T+1$ if it chooses to use Project E. We use a discount rate of $\alpha=0.99$ a the penalty cost of $\pi=\$ 20$ million per period.

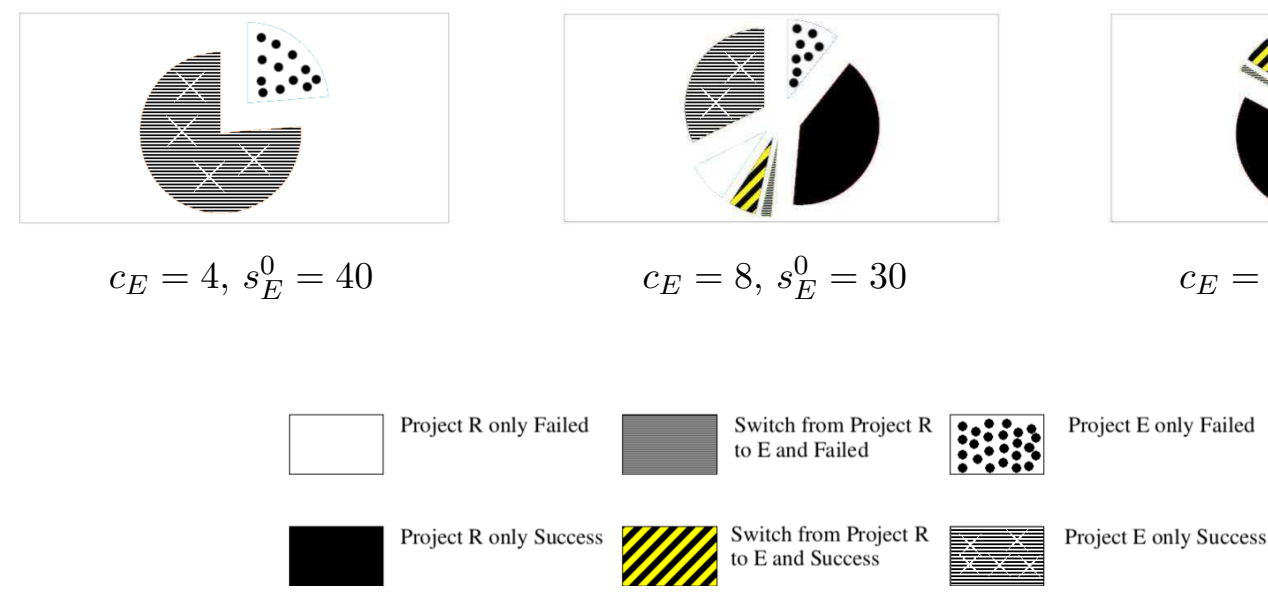

Figure 9: The choices between Project $\mathrm{R}$ and Project E under various parameter settings.

The pie charts in Figure 9 illustrate the optimal decisions under various cost parameters. In each pie chart, orange and light blue slices represent the proportion of sample paths that the firm chooses Project E only and the trial turns out positive or negative; the red and dark blue slices represent represent the proportion of sample paths that the firm chooses Project $\mathrm{R}$ only and the trial turns out positive or negative; and the purple and green slices represents the proportion of paths where firm first chooses Project $\mathrm{R}$ and switch to Project $\mathrm{E}$ and the trial turns out positive or negative. Note that there is no sample path where the firm switches from Project E to Project R.

We see that when $c_{E}=\$ 4$ million, so that the expedited project is relatively inexpensive (although always more expensive than the regular project), it dominates the regular project in all cases. However, as the cost of the expedited project increases, the expedited project is preferred less - the firm chooses between the two projects a bout evenly when Project E costs $c_{E}=\$ 8$ million per period and takes 30 periods to build; and when Project E costs the firm 20 periods and $c_{E}=\$ 16$ million per period, Project $\mathrm{R}$ acts as a backup alternative.

Indeed, the role of the expedited capacity changes as a function of its cost. When its cost is relatively low, the firm tends to wait for more information and to build the expedited capacity rather than the regular capacity, enabling it to limit its "wasted" construction. However, as the 
cost of expedited capacity increases, the firm increasingly uses expedited capacity as a backup to make up "lost time" after construction of the original cheaper capacity is stopped. In other words, when the expedited capacity is relatively cheap, the firm is better off paying for this expedited capacity to "buy" more time for information updates. When $c_{E}=\$ 4$ and the product ultimately fails, $73 \%$ of the time the firm makes the correct decision not to build any capacity. In contrast, when $c_{E}=\$ 16$, the firm is much more likely to build capacity early in the horizon, and so invests in regular capacity $13 \%$ of the time when there ultimately isn't any demand.

\section{Conclusion and Future Research}

In this paper, we studied the capacity investment problem faced by a firm that requires new production capacity for a product that is still undergoing testing. We are motivated by the pharmaceutical industry, where firms, under pressure to recover $R \& D$ costs as quickly as possible, traditionally make a one-time commitment to build or acquire production capacity so that the new drug can be launched on time. However, it is typically difficult to gauge the likelihood of passing required clinical trials early in the process. We developed a stylized model to study the value of periodically reviewing data from the on-going clinical trial and appropriately adjusting the ongoing capacity acquisition project, and characterized the optimal building strategy under various model settings. We used a computational study to explore the value of more frequently evaluating investments decisions, of accounting for the fact that information improves over time, of stopping and starting investments as information improves, and of investing in alternative types of capacity, as well as the impact of fixed costs and increasing marginal penalty costs. Our computational study suggests that in many cases, reevaluation dramatically reduces both the total expected investment cost, as well as the costs associated with the "mistakes" made in building too early or too late, i.e., the investment cost wasted in case of a failed clinical trial and the penalty cost of delayed production.

Of course, this stylized model significantly simplifies both capacity acquisition and clinical trial data collection. We are currently working to address several key simplifications. Our model implicitly assumes that the arrival rate of patients to the trial (and thus, the rate that results are available) is known - we make this assumption for analytical tractability, and it does model some classes of clinical trials, but sometimes, although the desired sample size is known, the arrival rate of patients to the trial (that is, the rate that patients with particular symptoms can be found and enrolled) is difficult to predict, particularly for uncommon diseases or symptoms. Also, we assume for analytical tractability that capacity investments are linear over time - we make this 
assumption for analytical tractability, and for some types of capacity investments it is appropriate, but obviously in some cases capacity investment is back-loaded or front-loaded. Finally, as we alluded to earlier, firms typically are not considering a capacity expansion project in a vacuum - they are typically optimizing a portfolio of capacity options at different stages of construction, and a portfolio of drugs at different stages in the pipeline, and the analysis of these portfolios is a promising area of research.

Nevertheless, we believe that the key takeaways from this research will hold in more complex settings: the value of regularly reviewing clinical data and reassessing ongoing capacity investment projects, the impact of available information and cost structure on this value, and the marginal value of increasing the frequency of reassessment of new information in various settings. Moreover, our computational study generated insights into the value of alternative expedited capacity of the type that is increasingly being considered in the biopharmaceutical industry.

\section{References}

Azoury, K. S. (1985). Bayes solution to dynamic inventory models under unknown demand distribution. Management Science, 31(9):pp. 1150-1160.

Bean, J. C., Higle, J. L., and Smith, R. L. (1992). Capacity expansion under stochastic demands. Operations Research, 40:pp. S210-S216.

Braden, D. J. and Freimer, M. (1991). Informational dynamics of censored obeservations. Management Science, 37(11):pp. 1390-1404.

Carroll, J. (2012a). In fresh setback, Alnylam's most advanced drug flunks PhIIb RSV test. http://www.fiercebiotech.com/story/ fresh-setback-alnylams-most-advanced-drug-flunks-phiib-rsv-test/2012-05-30.

Carroll, J. (2012b). Repligen shares slide after FDA rejects imaging agent. http://www. fiercebiotech.com/story/repligen-shares-slide-after-fda-rejects-imaging-agent/ 2012-06-22.

Chao, X., Chen, H., and Zheng, S. (2009). Dynamic capacity expansion for a service firm with capacity deterioration and supply uncertainty. Operations Research, 57(1):pp. 82-93.

Chod, J. and Rudi, N. (2006). Strategic investments, trading, and pricing under forecast updating. Management Science, 52(12):pp. 954-971.

Davis, M. H. A., Dempster, M. A. H., Sethi, S. P., and Vermes, D. (1987). Optimal capacity expansion under uncertainty. Advances in Applied Probability, 19(1):pp. 156-176.

DiMasi, J. A., Hansen, R. W., and Grabowski, H. G. (2003). The price of innovation: new estimates of drug development costs. Journal of Health Economics, 22:151-185.

Dixit, A. K. and Pindyck, R. S. (2004). Investment Under Uncertainty. Princeton University Press.

Elsanosi, I., Oksendal, B., and Sulem, A. (2000). Some solvable stochastic control problems with delay. Stochastics and Stochastics Reports, 71:pp. 69-89.

Erlenkotter, D. (1977). Capacity expansion with imports and inventories. Management Science, 23(7):pp. 694-702. 
Farid, S. S., Washbrook, J., and Titchener-Hooker, N. J. (2005). Decision-support tool for assessing biomanufacturing strategies under uncertainty: Stainless steel versus disposable equipment for clinical trial material preparation. Biotechnology Progress, 21(2):486-497.

Friedman, L. M., Furberg, C. D., and DeMets, D. L. (2010). Fundamentals of Clinical Trials. Springer, 4th ed. edition.

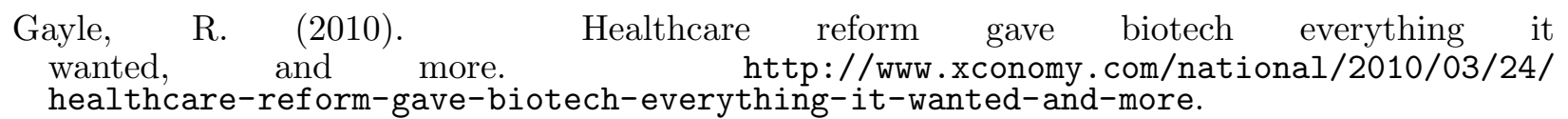

Genentech (2009). Genentech web site, manufacturing, accessed 11/1/2009. http://www.gene . com/gene/news/kits/corporate/manufacturing.html.

George, E., Titchener-Hooker, N. J., and Farid, S. S. (2007). A multi-criteria decision-making framework for the selection of strategies for acquiring biopharmaceutical manufacturing capacity. Computers 85 Chemical Engineering, 31(8):889 - 901. 7th World Congress of Chemical Engineering.

Gold, E. R., Castle, D., Cloutier, L. M., Daar, A. S., and Smith, P. J. (2002). Needed: models of biotechnology intellectual property. Trends in Biotechnology, 20(8):pp. 327-329.

Lakhdar, K., Zhou, Y., Savery, J., Titchener-Hooker, N. J., and Papageorgiou, L. G. (2005). Medium term planning of biopharmaceutical manufacture using mathematical programming. Biotechnology Progress, 21(5):1478-1489.

Lariviere, M. A. and Porteus, E. L. (1999). Bayesian inventory management with unobserved lost sales. Management Science, 45(3):pp. 346-363.

Luss, H. (1982). Operations research and capacity expansion problems: A survey. Operations Research, 30(5):pp. 907-947.

Manne, A. S. (1961). Capacity expansion and probabilistic growth. Econometrica, 29(4):pp. 632649 .

Morrow, K. J. (2006). Disposable bioreactors gaining favour: New components and systems improve process reliability and reduce cost. Naval Research Logistics (NRL), 26(12):42-45.

Papageorgiou, L. G., Rotstein, G. E., and Shah, N. (2001). Strategic supply chain optimization for the pharmaceutical industries. Industrial 85 Engineering Chemistry Research, 40(1):275-286.

Plambeck, E. L. and Taylor, T. A. (2005). Sell the plant? the impact of contract manufacturing on innovation, capacity, and proertability. Management Science, 51(1):pp. 133-150.

PMLive (2012). Amgen's Mimpara disappoints in phase III study. http://www.pmlive.com/ pharma_news/amgen_mimpara_disappoints_phase_iii_study_407263.

Robbins, H. (1952). Some aspects of the sequential design of experiments. Bulletin of the American Mathematical Society, 55:pp. 527-535.

Ryan, S. M. (2004). Capacity expansion for random exponential demand growth with lead times. Management Science, 50(6):pp. 740-748.

Scarf, H. (1959). Bayes solutions of the statistical inventory problem. The Annals of Mathematical Statistics, 30(2):pp. 490-508.

Sinden, F. W. (1960). The replacement and expansion of durable equipment. Journal of the Society for Industrial and Applied Mathematics, 8(3):pp. 466-480.

Smith, R. L. (1979). Turnpike results for single location capacity expansion. Management Science, 25(5):pp. 474-484. 
Snow, D. C., Wheelwright, S. C., and Wagonfeld, A. B. (2005). Genentech - capacity planning. Harvard Business.

Trigeorgis, L. (1996). Real Options: Managerial Flexibility and Strategy in Resource Allocation. MIT Press.

Van Mieghem, J. A. (1999). Coordinating investment, production, and subcontracting. Management Science, 45(7):pp. 954-971.

Van Mieghem, J. A. (2003). Capacity management, investment, and hedging: Review and recent developments. Manufacturing \& Service Operations Management, 5(4):pp. 267-302. $\begin{array}{ccc}\text { Xcellerex (2012). } & \text { Single-use bioreactor. } & \text { http://www.xcellerex.com/ } \\ \text { platform-xdr-single-use-bioreactors.htm. } & \end{array}$ 


\section{Online Supplement for}

Production Capacity Investment with Data Updates

by Philip Kaminsky and Ming Yuen

\section{A Proof of Lemma 4.1:}

By direct calculation,

$$
\begin{aligned}
\operatorname{Pr}\left(\sum_{j=1}^{m} \delta^{t-1+j}=k \mid \gamma^{t-1}, \gamma^{t-2}, \ldots, \gamma^{0}\right) & =\left(\begin{array}{c}
m \\
k
\end{array}\right) \frac{\Gamma\left(\gamma^{t-1}+\zeta^{t-1}\right)}{\Gamma\left(\gamma^{t-1}\right) \Gamma\left(\zeta^{t-1}\right)} \int_{0}^{1} p^{\gamma^{t-1}+k-1}(1-p)^{\zeta^{t-1}+(m-k)-1} d p \\
& =\left(\begin{array}{c}
m \\
k
\end{array}\right) \frac{\prod_{j=0}^{k-1}\left(\gamma^{t-1}+j\right) \prod_{j=0}^{m-k-1}\left(\zeta^{t-1}+j\right)}{\prod_{j=0}^{m-1}\left(\gamma^{t-1}+\zeta^{t-1}+j\right)} \\
& =\operatorname{Pr}\left(\sum_{j=1}^{m} \delta^{t-1+j}=k \mid \gamma^{t-1}\right)
\end{aligned}
$$

\section{B Proof of Lemma 4.2:}

$$
\operatorname{Pr}\left(\gamma^{T} \geq \gamma^{c} \mid \gamma^{t-1}=\gamma\right)=\frac{\sum_{k=\left(\gamma^{c}-\gamma\right)^{+}}^{T-t+1}\left(\begin{array}{c}
T-t+1 \\
k
\end{array}\right) \prod_{j=0}^{k-1}\left(\gamma^{t-1}+j\right) \prod_{j=0}^{T-t-k}\left(\zeta^{t-1}+j\right)}{\prod_{j=0}^{T-t}\left(\gamma^{t-1}+\zeta^{t-1}+j\right)} .
$$

With some algebraic manipulation, we have:

$$
\begin{aligned}
& \operatorname{Pr}\left(\gamma^{T} \geq \gamma^{c} \mid \gamma^{t-1}=\gamma\right)-\operatorname{Pr}\left(\gamma^{T} \geq \gamma^{c} \mid \gamma^{t-1}=\gamma-1\right) \\
& =\frac{\sum_{k=\left(\gamma^{c}-\gamma\right)^{+}}^{T-t}\left(\begin{array}{c}
T-t+1 \\
k
\end{array}\right) \prod_{j=0}^{k-1}\left(\gamma^{t-1}+j\right) \prod_{j=1}^{T-i-k}\left(\zeta^{t-1}+j\right)\left[\zeta^{t-1}-\left(\gamma^{t-1}-1\right) \frac{T-t-k+1}{k+1}\right]+\prod_{j=0}^{T-t}\left(\gamma^{t-1}+j\right)}{\prod_{j=0}^{T-t}\left(\gamma^{t-1}+\zeta^{t-1}+j\right)} .
\end{aligned}
$$


Note that the term

$$
\left(\zeta^{t-1}\right)(k+1)-\left(\gamma^{t-1}-1\right)(T-t-k+1)
$$

is increasing in $k$, and changes sign at most once. Combined with the fact that all other terms remain positive, we observe that:

$$
\begin{aligned}
& \operatorname{Pr}\left(\gamma^{T} \geq \gamma^{c} \mid \gamma^{t-1}=\gamma\right)-\operatorname{Pr}\left(\gamma^{T} \geq \gamma^{c} \mid \gamma^{t-1}=\gamma-1\right) \\
\geq & \min \left\{\operatorname{Pr}\left(\gamma^{T} \geq \gamma^{c} \mid \gamma^{t-1}=\gamma^{c}\right)-\operatorname{Pr}\left(\gamma^{T} \geq \gamma^{c} \mid \gamma^{t-1}=\gamma^{c}-1\right),\right. \\
& \left.\operatorname{Pr}\left(\gamma^{T} \geq \gamma^{c} \mid \gamma^{t-1}=\gamma^{c}-T+t-1\right)-\operatorname{Pr}\left(\gamma^{T} \geq \gamma^{c} \mid \gamma^{t-1}=\gamma^{c}-T+t-2\right)\right\}
\end{aligned}
$$

$\geq 0$,

and by transitivity, we have:

$$
\operatorname{Pr}\left(\gamma^{T} \geq \gamma^{c} \mid \gamma^{t-1}=\gamma_{1}\right) \geq \operatorname{Pr}\left(\gamma^{T} \geq \gamma^{c} \mid \gamma^{t-1}=\gamma_{2}\right)
$$

for all $\gamma_{1}>\gamma_{2}$.

\section{Proof of Lemma 4.3:}

From (4.1), we show that $\gamma^{t-1}$ is a Markov process, and by conditioning on $\gamma^{t}$, we have:

$$
\begin{aligned}
& \operatorname{Pr}\left(\gamma^{T} \geq \gamma^{c} \mid \gamma^{t}=\gamma+1\right)-\operatorname{Pr}\left(\gamma^{T} \geq \gamma^{c} \mid \gamma^{t-1}=\gamma\right) \\
= & \left.\operatorname{Pr}\left(\gamma^{T} \geq \gamma^{c} \mid \gamma^{t}=\gamma+1\right)-\sum_{i=0}^{1} \operatorname{Pr}\left(\gamma^{T} \geq \gamma^{c} \mid \gamma^{t}=\gamma+i\right)\right] \operatorname{Pr}\left(\delta^{t}=i \mid \gamma^{t-1}=\gamma\right) \geq 0
\end{aligned}
$$

The inequality results from a direct application of Lemma 4.2.

\section{Proof of Lemma 4.4:}

Similar to the proof of Lemma 4.3, we have:

$$
\begin{aligned}
& \operatorname{Pr}\left(\gamma^{T} \geq \gamma^{c} \mid \gamma^{t}=\gamma\right)-\operatorname{Pr}\left(\gamma^{T} \geq \gamma^{c} \mid \gamma^{t-1}=\gamma\right) \\
= & \operatorname{Pr}\left(\gamma^{T} \geq \gamma^{c} \mid \gamma^{t}=\gamma\right)-\sum_{i=0}^{1} \operatorname{Pr}\left(\gamma^{T} \geq \gamma^{c} \mid \gamma^{t}=\gamma+i\right) \operatorname{Pr}\left(\delta^{t}=i \mid \gamma^{t-1}=\gamma\right) \leq 0 .
\end{aligned}
$$


The inequality again results from a direct application of Lemma 4.2.

\section{E Proof of Lemma 5.1:}

Suppose $T>s_{\max }^{0}$ and consider an admissible strategy $\mathbf{a}^{\mathbf{1}}$ as a potential candidate optimal strategy at period $t=1$ with $\mathbf{a}^{\mathbf{1}} \neq 0$. We define index $I^{\prime}$ to identify the entries such that $a_{i}^{1}=1$. We further define a stopping time for each $i \in I^{\prime}, \tau_{i}:=\min \left\{t \mid a_{i}^{t}=0\right\}$, and note that $\tau \leq s_{\max }^{0}<T$ a.s., since it is a waste of resources to invest in a project which is already completed. We consider an alternative strategy $\mathbf{a}^{\mathbf{1}}$ such that $\mathbf{a}^{\mathbf{1}}=0$, and for $t=2, \ldots, T$. We couple $\mathbf{a}^{\text {t }}$ with $\mathbf{a}^{\mathbf{t}}$ for each outcome $\gamma_{t-1}$, such that $a_{i}^{\prime t}=a_{i}^{t}$ for $i \in I \backslash I^{\prime}$ and for each $t=2,3, \ldots, T$. For facility types $i \in I^{\prime}$, we couple $a_{i}^{\prime t}$ with $a_{i}^{t}$ such that,

$$
a_{i}^{\prime t}=\left\{\begin{array}{cc}
1 & \text { for } t=\tau_{i}, \\
a_{i}^{t} & \text { otherwise }
\end{array}\right.
$$

Let $J\left(\gamma_{0}, \mathbf{s}^{\mathbf{0}}, \mathbf{a}^{\mathbf{0}}, \mathbf{a}^{\mathbf{1}}\right)$ be the expected total cost if the firm uses investment strategy $\mathbf{a}^{\mathbf{1}}$ at period $t=1$, then

$$
\begin{aligned}
J\left(\gamma_{0}, \mathbf{s}^{\mathbf{0}}, \mathbf{a}^{\mathbf{0}}, \mathbf{a}^{\mathbf{1}}\right) & \geq J\left(\gamma_{0}, \mathbf{s}^{\mathbf{0}}, \mathbf{a}^{\mathbf{0}}, \mathbf{a}^{\mathbf{1}}\right)+E\left[\sum_{i \in I^{\prime}} \sum_{j=1}^{\tau_{i}}\left(\alpha^{j-1}-\alpha^{j}\right) C_{i}\left(s_{i}^{0}-j+1\right) \mid \gamma_{0}\right] \\
& \geq J\left(\gamma_{0}, \mathbf{s}^{\mathbf{0}}, \mathbf{a}^{\mathbf{0}}, \mathbf{a}^{\mathbf{1}}\right) \\
& \geq V_{1}\left(\gamma_{0}, \mathbf{s}^{\mathbf{0}}, \mathbf{a}^{\mathbf{0}}\right) .
\end{aligned}
$$

The decision, $\mathbf{a}^{\mathbf{t}}$, indeed gives a suboptimal solution at $t=1$, therefore by contradiction,

$$
V_{1}\left(\gamma^{0}, \mathbf{s}^{\mathbf{0}}, \mathbf{a}^{\mathbf{0}}\right)=\alpha E\left[V_{2}\left(\gamma^{1}, \mathbf{s}^{\mathbf{0}}, \mathbf{a}^{\mathbf{0}}\right) \mid \gamma^{0}\right]
$$

\section{F Proof of Theorem 5.2:}

The result is a consequence of Lemma 4.2 and (10). 


\section{G Proof of Corollary 5.3:}

From Lemma 4.2, and the monotonicity stated in Theorem 5.2:

$$
\operatorname{Pr}\left(\gamma^{T} \geq \gamma^{c} \mid \gamma^{t}=\gamma+1\right) \geq \operatorname{Pr}\left(\gamma^{T} \geq \gamma^{c} \mid \gamma^{t-1}=\gamma\right) \geq \operatorname{Pr}\left(\gamma^{T} \geq \gamma^{c} \mid \gamma^{t-1}=\gamma_{*}^{t-1}\right) \geq \frac{c}{\alpha^{T-t}(c+\pi)}
$$

therefore, it is also optimal for the firm to invest in period $t$.

Proof of Corollary 5.4: The corollary is a direct consequence of Theorem 5.2 and Lemma 4.4.

\section{H Proof of Theorem 5.5:}

Here, we observe that the firm should build capacity for period $t$ if:

$$
\operatorname{Pr}\left(\gamma^{T} \geq \gamma^{c} \mid \gamma^{t-1}\right) \geq \frac{c+K+\alpha E\left[W_{t+1}\left(\gamma^{t}, s^{t-1}-1\right) \mid \gamma^{t-1}\right]}{\alpha^{T-t+1}(c+\pi)}
$$

when $a^{t-1}=0$; and

$$
\operatorname{Pr}\left(\gamma^{T} \geq \gamma^{c} \mid \gamma^{t-1}\right) \geq \frac{c+\alpha E\left[W_{t+1}\left(\gamma^{t}, s^{t-1}-1\right) \mid \gamma^{t-1}\right]}{\alpha^{T-t+1}(c+\pi)}
$$

when $a^{t-1}=1$. Since $K>0$, if we can show that for $W(\cdot, \cdot)$ as defined in equation (11), $E\left[W_{t+1}\left(\gamma^{t}, s^{t-1}-1\right) \mid \gamma^{t-1}\right]$ is a non-increasing function in $\gamma^{t-1}$ for all $t$, then for each $t$ there exist a pair of investment thresholds $\gamma_{*_{0}}^{t-1} \geq \gamma_{*_{1}}^{t-1}$,

$$
\begin{aligned}
& \gamma_{*_{0}}^{t-1}:=\inf \left\{\gamma \in\left\{\gamma^{0}, \ldots, \gamma^{0}+t-1\right\} \mid \operatorname{Pr}\left(\gamma^{T} \geq \gamma^{c} \mid \gamma^{t-1}=\gamma\right) \geq \frac{c+K+\alpha E\left[W_{t+1}\left(\gamma^{t}, s^{t-1}-1\right) \mid \gamma^{t}\right]}{\alpha^{T-t+1}(c+\pi)}\right\}, \\
& \gamma_{*_{1}}^{t-1}:=\inf \left\{\gamma \in\left\{\gamma^{0}, \ldots, \gamma^{0}+t-1\right\} \mid \operatorname{Pr}\left(\gamma^{T} \geq \gamma^{c} \mid \gamma^{t-1}=\gamma\right) \geq \frac{c+\alpha E\left[W_{t+1}\left(\gamma^{t}, s^{t-1}-1\right) \mid \gamma^{t}\right]}{\alpha^{T-t+1}(c+\pi)}\right\},
\end{aligned}
$$

such that for $a^{t-1}=0$ the firm should built at period $t$ if and only if $\gamma^{t-1} \geq \gamma_{*_{0}}^{t-1}$; and for $a^{t-1}=1$ if and only if $\gamma^{t-1} \geq \gamma_{*_{1}}^{t-1}$. To show $E\left[W_{t+1}\left(\gamma^{t}, s^{t-1}\right) \mid \gamma^{t-1}\right]$ is non-increasing in $\gamma^{t-1}$ for all $t \in\{1,2, \ldots, T+1\}$, we use induction. For $t=T+1$,

$$
W_{T+1}\left(\gamma^{T}, s^{T}\right)= \begin{cases}0 & \text { for } \gamma^{T}<\gamma^{c} \\ -K & \text { for } \gamma^{T} \geq \gamma^{c}\end{cases}
$$


Then by Lemma 4.2 , we see $E\left[W_{T+1}\left(\gamma^{T}, s^{T}\right) \mid \gamma^{T-1}\right]$ is non-increasing in $\gamma^{T-1}$, and $\gamma_{*_{0}}^{T-1} \geq \gamma_{*_{1}}^{T-1}$. Now we suppose $E\left[W_{t+1}\left(\gamma^{t}, s^{t}\right) \mid \gamma^{t-1}\right]$ is non-increasing in $\gamma^{t-1}$ at period $t$ and need to show it is also true for period $t-1$. Observe:

$W_{t}\left(\gamma^{t-1}, s^{t-1}\right)= \begin{cases}0 & \text { for } \gamma^{t-1}<\gamma_{*_{1}}^{t-1}, \\ c+\alpha E\left[W_{t+1}\left(\gamma^{t}, s^{t}\right) \mid \gamma^{t-1}\right]-\alpha^{T-t+1}(c+\pi) \operatorname{Pr}\left(\gamma^{T} \geq \gamma^{c} \mid \gamma^{t-1}\right) & \text { for } \gamma_{*_{1}}^{t-1} \leq \gamma^{t-1} \leq \gamma_{*_{0}}^{t-1} \\ -K & \text { for } \gamma^{t-1} \geq \gamma_{*_{0}}^{t-1}\end{cases}$

By Lemma $4.2, E\left[W_{t}\left(\gamma^{t-1}, s^{t-1}\right) \mid \gamma^{t-2}\right]$ is non-increasing in $\gamma^{t-2}$.

\section{Proof of Corollary 5.6:}

Recall

$$
\begin{gathered}
\gamma_{*_{0}}^{t-1}:=\inf \left\{\gamma \in \Gamma \mid \operatorname{Pr}\left(\gamma^{T} \geq \gamma^{c} \mid \gamma^{t-1}=\gamma\right) \geq \frac{c+K+\alpha E\left[W_{t+1}\left(\gamma^{t}, s^{t-1}-1\right) \mid \gamma^{t-1}\right]}{\alpha^{T-t+1}(c+\pi)}\right\}, \\
\gamma_{*_{1}}^{t-1}:=\inf \left\{\gamma \in \Gamma \mid \operatorname{Pr}\left(\gamma^{T} \geq \gamma^{c} \mid \gamma^{t-1}=\gamma\right) \geq \frac{c+\alpha E\left[W_{t+1}\left(\gamma^{t}, s^{t-1}-1\right) \mid \gamma^{t-1}\right]}{\alpha^{T-t+1}(c+\pi)}\right\}, \text { and } \\
\gamma_{*}^{t-1}:=\inf \left\{\gamma \in \Gamma \mid \operatorname{Pr}\left(\gamma^{T} \geq \gamma^{c} \mid \gamma^{t-1}=\gamma\right) \geq \frac{c}{\alpha^{T-t+1}(c+\pi)}\right\} .
\end{gathered}
$$

From the proof of Theorem 5.5, we observe

$$
-K<E\left[W_{t+1}\left(\gamma^{t}, s^{t-1}-1\right) \mid \gamma^{t-1}\right]<0
$$

and $\operatorname{Pr}\left(\gamma^{T} \geq \gamma^{c} \mid \gamma^{t-1}=\gamma\right)$ is monotonic increasing in $\gamma$. Therefore,

$$
\gamma_{*_{1}}^{t-1} \leq \gamma_{*}^{t-1} \leq \gamma_{*_{0}}^{t-1}
$$

\section{J Proof of Theorem 5.7:}

Note that since the setup cost is zero, we rewrite the penalty function as follows:

$$
F\left(s^{T}, a^{T}\right)=F\left(s^{T}\right)
$$


The proof proceeds by backward induction:

i. Suppose period $t=T$,

$$
\begin{aligned}
& V_{T}\left(\gamma^{T-1}, s^{T-1}\right) \\
= & \min \left\{c, \alpha\left(F\left(s^{T-1}\right)-F\left(s^{T-1}-1\right)\right) \operatorname{Pr}\left(\gamma^{T} \geq \gamma^{c} \mid \gamma^{T-1}\right)\right\}+\alpha F\left(s^{T-1}-1\right) \operatorname{Pr}\left(\gamma^{T} \geq \gamma^{c} \mid \gamma^{T-1}\right) .
\end{aligned}
$$

By Lemma 4.2, there exists $\gamma_{*_{s}}^{T-1}$ for each $s^{T-1}=s$ such that the firm should invest if and only if $\gamma^{T-1} \geq \gamma_{*_{s}}^{T-1}$.

ii. Moreover, by $(12), \gamma_{*_{s}}^{T-1}$ is decreasing in $s$.

iii. After some algebraic manipulation, we have:

$$
\begin{aligned}
& V_{T}\left(\gamma^{T-1}, s\right)-V_{T}\left(\gamma^{T-1}, s-1\right) \\
= & \begin{cases}\alpha(F(s-1)-F(s-2)) \operatorname{Pr}\left(\gamma^{T} \geq \gamma^{c} \mid \gamma^{T-1}\right) & \text { for } \gamma^{T-1} \geq \gamma_{*_{s-1}}^{T-1}, \\
c & \text { for } \gamma_{*_{s}}^{T-1} \leq \gamma^{T-1}<\gamma_{*_{s-1}}^{T-1}, \quad \geq 0 . \\
\alpha(F(s)-F(s-1)) \operatorname{Pr}\left(\gamma^{T} \geq \gamma^{c} \mid \gamma^{T-1}\right) & \text { for } \gamma^{T-1}<\gamma_{*_{s}}^{T-1} .\end{cases}
\end{aligned}
$$

By Lemma 4.2, we also see that $V_{T}\left(\gamma^{T-1}, s\right)-V_{T}\left(\gamma^{T-1}, s-1\right)$ is increasing in $\gamma^{T-1}$.

iv. For the convexity of $V_{T}\left(\gamma^{T-1}, s\right)$ in $s$, we observe:

$$
\begin{aligned}
& V_{T}\left(\gamma^{T-1}, s+1\right)-2 V_{T}\left(\gamma^{T-1}, s\right)+V_{T}\left(\gamma^{T-1}, s-1\right) \\
= & \begin{cases}\alpha[F(s)-2 F(s-1)+F(s-2)] \operatorname{Pr}\left(\gamma^{T} \geq \gamma^{c} \mid \gamma^{T-1}\right) & \text { for } \gamma^{T-1} \geq \gamma_{*_{s}-2}^{T-1}, \\
\alpha(F(s)-F(s-1)) \operatorname{Pr}\left(\gamma^{T} \geq \gamma^{c} \mid \gamma^{T-1}\right)-c & \text { for } \gamma_{*_{s-1}}^{T-1} \leq \gamma^{T-1}<\gamma_{*_{s-2}}^{T-1}, \\
c-\alpha(F(s)-F(s-1)) \operatorname{Pr}\left(\gamma^{T} \geq \gamma^{c} \mid \gamma^{T-1}\right) & \text { for } \gamma_{*_{s}}^{T-1} \leq \gamma^{T-1}<\gamma_{*_{s-1}}^{T-1}, \\
\alpha[F(s+1)-2 F(s)+F(s-1)] \operatorname{Pr}\left(\gamma^{T} \geq \gamma^{c} \mid \gamma^{T-1}\right) & \text { for } \gamma^{T-1}<\gamma_{*_{s}}^{T-1} .\end{cases}
\end{aligned}
$$

Therefore, $V_{T}\left(\gamma^{T-1}, s\right)$ is convex in $s$.

Now we suppose that the above statements i, ii, iii and iv hold true for $t+1$ and show the they hold true for $t$. Observe:

$$
V_{t}\left(\gamma^{t-1}, s\right)=\min \left\{c, \alpha E\left[V_{t+1}\left(\gamma^{t}, s\right)-V_{t+1}\left(\gamma^{t}, s-1\right) \mid \gamma^{t-1}\right]\right\}+\alpha E\left[V_{t+1}\left(\gamma^{t}, s-1\right) \mid \gamma^{t-1}\right]
$$

- By Lemma 4.2 and $V_{t+1}\left(\gamma^{t}, s\right)-V_{t+1}\left(\gamma^{t}, s-1\right)$ is increasing in $\gamma^{t}$. Therefore, we conclude that there exists a $\gamma_{*_{s}}^{t-1}$ for each $s^{t-1}=s$, such that the firm should invest if and only if 


$$
\gamma^{t-1} \geq \gamma_{*_{s}}^{t-1}
$$

- In addition, by the convexity of $V_{t+1}\left(\gamma^{t}, s\right)$ in $s$, we see that $\gamma_{*_{s}}^{t-1}$ is decreasing in $s$.

- Again, after algebraic manipulation, we have:

$$
\begin{aligned}
& V_{t}\left(\gamma^{t-1}, s\right)-V_{t}\left(\gamma^{t-1}, s-1\right) \\
= & \begin{cases}E\left[V_{t+1}\left(\gamma^{t}, s-1\right)-V_{t+1}\left(\gamma^{t}, s-2\right) \mid \gamma^{t-1}\right] & \text { for } \gamma^{t-1} \geq \gamma_{*_{s-1}}^{t-1}, \\
c & \text { for } \gamma_{*_{s}}^{t-1} \leq \gamma^{t-1}<\gamma_{*_{s-1}}^{t-1}, \quad \geq 0 . \\
E\left[V_{t+1}\left(\gamma^{t}, s\right)-V_{t+1}\left(\gamma^{t}, s-1\right) \mid \gamma^{t-1}\right] & \text { for } \gamma^{t-1}<\gamma_{*_{s}}^{t-1} .\end{cases}
\end{aligned}
$$

- Then by Lemma 4.2, we see that $V_{t}\left(\gamma^{t-1}, s\right)-V_{t}\left(\gamma^{t-1}, s-1\right)$ is increasing in $\gamma^{t-1}$.

- For the convexity of $V_{t}\left(\gamma^{t-1}, s\right)$ in $s$ :

$$
\begin{aligned}
& V_{t}\left(\gamma^{t-1}, s+1\right)-2 V_{t}\left(\gamma^{t-1}, s\right)+V_{t}\left(\gamma^{t-1}, s-1\right) \\
= & \begin{cases}\alpha E\left[V_{t+1}\left(\gamma^{t}, s\right)-2 V_{t+1}\left(\gamma^{t} s-1\right)+V_{t+1}\left(\gamma^{t}, s-2\right) \mid \gamma^{t-1}\right] & \text { for } \gamma^{t-1} \geq \gamma_{*_{s-2}}^{t-1}, \\
\alpha E\left[V_{t+1}\left(\gamma^{t}, s-1\right)-V_{t+1}\left(\gamma^{t}, s-2\right) \mid \gamma^{t-1}\right]-c & \text { for } \gamma_{*_{s-1}}^{t-1} \leq \gamma^{-1 t}<\gamma_{*_{s-2}}^{t-1}, \quad \geq 0 . \\
c-\alpha E\left[V_{t+1}\left(\gamma^{t}, s-1\right)-V_{t+1}\left(\gamma^{t}, s-2\right) \mid \gamma^{t-1}\right] & \text { for } \gamma_{*_{s}}^{t-1} \leq \gamma^{t-1}<\gamma_{*_{s-1}}^{t-1}, \\
\alpha E\left[V_{t+1}\left(\gamma^{t}, s+1\right)-2 V_{t+1}\left(\gamma^{t}, s\right)+V_{t+1}\left(\gamma^{t}, s-1\right) \mid \gamma^{t-1}\right] & \text { for } \gamma^{t-1}<\gamma_{*_{s}}^{t-1} .\end{cases}
\end{aligned}
$$

\section{K Proof of Lemma 5.8:}

Here, we calculate $k_{1}^{t}$ and $k_{2}^{t}$ directly. At $t=T+1$, we see that $k_{1}^{T+1}=k_{2}^{T+1}=0$ from the penalty function (13).

At $t=T$, for $s_{E} \geq \frac{s_{R}\left(\pi+c_{R}\right)-O}{\pi+c_{E}}+1$, since $E\left[V_{T+1}\left(\gamma^{T}, s_{R}, s_{E}-1\right) \mid \gamma^{T-1}\right]=E\left[V_{T+1}\left(\gamma^{T}, s_{R}, s_{E}\right) \mid \gamma^{T-1}\right]=$ $E\left[V_{T+1}\left(\gamma^{T}, s_{R}\right) \mid \gamma^{T-1}\right]$, it is not optimal for the firm to build Project E. Similarly, for $s_{E}<$ $\frac{\left(s_{R}-1\right)\left(\pi+c_{R}\right)-O}{\pi+c_{E}}$, it is not optimal for the firm to build Project R. For $\frac{s_{R}\left(\pi+c_{R}\right)-O}{\pi+c_{E}} \leq s_{E}<\frac{s_{R}\left(\pi+c_{R}\right)-O}{\pi+c_{E}}+$ 1, the firm should choose Project E over Project $R$ when

$$
s_{E} \geq \frac{s_{R}\left(\pi+c_{R}\right)-O}{\pi+c_{E}}-\frac{c_{E}-c_{R}}{\pi+c_{E}}\left(\frac{1}{\alpha \operatorname{Pr}\left(\gamma^{T} \geq \gamma^{c} \mid \gamma^{T-1}\right)}-1\right)
$$

However, since it is true when

$$
s_{E} \geq \frac{s_{R}\left(\pi+c_{R}\right)-O}{\pi+c_{E}}
$$


in this region, it is not optimal for the firm to build Project R. For $\frac{s_{R}\left(\pi+c_{R}\right)-O}{\pi+c_{E}}>s_{E} \geq \frac{\left(s_{R}-1\right)\left(\pi+c_{R}\right)-O}{\pi+c_{E}}$, the firm's decision is based on a three way comparison:

$$
\begin{array}{r}
\min \left\{\alpha\left[s_{E}\left(\pi+c_{E}\right)+O\right] \operatorname{Pr}\left(\gamma^{T} \geq \gamma^{c} \mid \gamma^{T-1}\right), c_{R}+\alpha\left(s_{R}-1\right)\left(\pi+c_{R}\right) \operatorname{Pr}\left(\gamma^{T} \geq \gamma^{c} \mid \gamma^{T-1}\right),\right. \\
\left.c_{E}+\alpha\left[\left(s_{E}-1\right)\left(\pi+c_{E}\right)+O\right] \operatorname{Pr}\left(\gamma^{T} \geq \gamma^{c} \mid \gamma^{T-1}\right)\right\},
\end{array}
$$

Note that, to choose Project R, we need:

$$
\left.\frac{C_{E}-C_{R}}{\alpha\left[\left(S_{R}-1\right)\left(\pi+C_{R}\right)-\left(S_{E}-1\right)\left(\pi+C_{E}\right)-O\right)} \geq \operatorname{Pr}\left(\gamma^{T} \geq \gamma^{c} \mid \gamma^{T-1}\right)\right]
$$

and

$$
\left.\frac{C_{R}}{\alpha\left[S_{E}\left(\pi+C_{E}\right)+O-\left(S_{R}-1\right)\left(\pi+C_{R}\right)\right)} \leq \operatorname{Pr}\left(\gamma^{T} \geq \gamma^{c} \mid \gamma^{T-1}\right)\right]
$$

However,

$$
\frac{c_{R}}{\alpha\left[s_{E}\left(\pi+c_{E}\right)+O-\left(s_{R}-1\right)\left(\pi+c_{R}\right)\right]} \leq \frac{c_{E}-c_{R}}{\alpha\left[\left(s_{R}-1\right)\left(\pi+c_{R}\right)-\left(s_{E}-1\right)\left(\pi+c_{E}\right)-O\right]},
$$

holds if and only if

$$
s_{E} \geq \frac{\left(s_{R}-1\right)\left(\pi+c_{R}\right)-O}{\pi+c_{E}}+\frac{c_{R}}{c_{E}}
$$

thus, it is not optimal for the firm to build Project $\mathrm{R}$ in the region $s_{E}<\frac{\left(s_{R}-1\right)\left(\pi+c_{R}\right)-O}{\pi+c_{E}}+\frac{c_{R}}{c_{E}}$. Therefore, $k_{1}^{T}=0$ and $k_{2}^{T}=\frac{c_{R}}{c_{E}}-\frac{\pi+c_{R}}{\pi-c_{E}}$.

At $t=T-1$, for $s_{E} \geq \frac{\left(s_{R}-1\right)\left(\pi+c_{R}\right)-O}{\pi+c_{E}}+\frac{c_{R}}{c_{E}}+1$, we have

$$
E\left[V_{T}\left(\gamma^{T-1}, s_{R}-1, s_{E}\right) \mid \gamma^{T-2}\right]=E\left[V_{T}\left(\gamma^{T-1}, s_{R}-1\right) \mid \gamma^{T-2}\right]=E\left[V_{T}\left(\gamma^{T-1}, s_{R}-1, s_{E}-1\right) \mid \gamma^{T-2}\right],
$$

therefore, it is not optimal for the firm to build Project E. Likewise, for $s_{E}<\frac{\left(s_{E}-2\right)\left(\pi+c_{R}\right)-O}{\pi+c_{E}}+\frac{c_{R}}{c_{E}}$, we have

$$
E\left[V_{T}\left(\gamma^{T-1}, s_{R}-1, s_{E}\right) \mid \gamma^{T-2}\right]=E\left[V_{T}\left(\gamma^{T-1}, s_{E}\right) \mid \gamma^{T-2}\right]=E\left[V_{T}\left(\gamma^{T-1}, s_{R}, s_{E}\right) \mid \gamma^{T-2}\right]
$$

the cost to go of building Project $\mathrm{R}$ is always greater than the cost of waiting, therefore, it is not optimal for the firm to build Project R. Thus, we have $k_{1}^{T-1}=\frac{c_{R}}{c_{E}}+\frac{c_{E}-c_{R}}{\pi+c_{E}}$ and $k_{2}^{T-1}=$ $\frac{c_{R}}{c_{E}}-2 \frac{\pi+c_{R}}{\pi+c_{E}}$ With similar analysis, we see that for $0 \leq t<T_{1}, k_{1}^{T-t}=\frac{c_{R}}{c_{E}}+(T-t) \frac{c_{E}-c_{R}}{\pi+c_{E}}$ and $k_{2}^{T-t}=\frac{c_{R}}{c_{E}}-(T-t+1) \frac{\pi+c_{R}}{\pi+c_{E}}$. 